\title{
A flow-condition-based interpolation finite element procedure for triangular grids $\ddagger$
}

\author{
Haruhiko Kohno and Klaus-Jürgen Bathe*, $\dagger$ \\ Department of Mechanical Engineering, Massachusetts Institute of Technology, 77 Massachusetts Avenue, \\ Cambridge, MA 02139-4307, U.S.A.
}

\begin{abstract}
SUMMARY
A flow-condition-based interpolation finite element scheme is presented for use of triangular grids in the solution of the incompressible Navier-Stokes equations. The method provides spatially isotropic discretizations for low and high Reynolds number flows. Various example solutions are given to illustrate the capabilities of the procedure. Copyright (c) 2006 John Wiley \& Sons, Ltd.
\end{abstract}

Received 7 March 2005; Revised 10 May 2005; Accepted 10 May 2005

KEY WORDS: incompressible flows; high Reynolds number flows; stability; accuracy; flow-conditionbased interpolation

\section{INTRODUCTION}

While much research has been expended on the numerical solution of the Navier-Stokes equations and certain computational schemes are in wide use, the more effective solution of general fluid flow problems at high Reynolds numbers still represents a major challenge, see References [1-4] and the many references therein.

In our research we have focused on the development of the flow-condition-based interpolation (FCBI) solution approach, which is a hybrid approach between the usual control volume and finite element methods, drawing on the best features of these techniques. The specific aim in the FCBI

\footnotetext{
${ }^{*}$ Correspondence to: K. J. Bathe, Department of Mechanical Engineering, Massachusetts Institute of Technology, 77 Massachusetts Avenue, Cambridge, MA 02139-4307, U.S.A.

†E-mail: kjb@mit.edu

$¥$ This article replaces a previously published version: International Journal for Numerical Methods in Fluids 2005; 49(8):849-875 [DOI: 10.1002/fld.1027].
}

Contract/grant sponsor: Ministry of Education, Culture, Sports, Science and Technology (MEXT), Japan 
solution approach is to reach procedures that are stable, accurate and efficient for any Reynolds number flow, even when rather coarse meshes are used for solution. The aims of our developments have been presented in detail in References [5-7].

In engineering practice, we endeavor to use as coarse meshes as possible for a required accuracy. Hence, we require a numerical solution procedure that is stable and gives reasonable solutions even when using rather coarse meshes for high Reynolds number flows. This numerical scheme should not require any special meshing directed to obtain a solution and not require the tuning of numerical parameters. Also, the iterations to solve the nonlinear algebraic equations corresponding to a fluid mesh should converge fast. Once a numerical solution-maybe even sometimes only of rough but still reasonable accuracy - has been reached, the analyst can refine the mesh in a targeted manner and change, appropriately, the mathematical modelling assumptions used (for example, regarding turbulence modelling).

The benefit of being able to use rather coarse meshes can be particularly pronounced in the analysis of fluid flow structural interactions, because in such analyses, actually, a rather coarse fluid flow mesh may well yield sufficient accuracy for the tractions on the structure [8]. Here then, in addition to obtaining sufficient accuracy in the fluid flow prediction, the iterations used to solve the combined nonlinear algebraic equations corresponding to the fluid flow and structural meshes should converge fast, and in many cases some Newton-Raphson procedure is best used with consistent Jacobian matrices [9].

The requirements that we have set for our developments within the FCBI solution approach are $[5-8,10]$ :

- Stability of the numerical solution for low and high Reynolds number flows, using coarse meshes. Reasonable accuracy of the solution.

- As the mesh is refined, stability is preserved and the accuracy of the simulation is optimally increased.

- The analyst does not use any numerical parameters to tune the fluid flow solution.

- The nonlinear algebraic equations can be solved efficiently in iterations using a consistent Jacobian matrix, say in the Newton-Raphson iterations (which requires that interpolations of the variables are used).

In our earlier contributions we presented FCBI schemes for quadrilateral grids, or general quadrilateral finite element meshes [5-7]. In practice, however, the use of triangular grids, and in three-dimensional analyses tetrahedral element meshes, is very desirable. Namely, any domain can be meshed with tetrahedral elements and for complicated geometries, tetrahedral element discretizations in unstructured meshes generally need to be used.

The objective in this paper is to present developments of an FCBI scheme using triangular grids for two-dimensional solutions of Navier-Stokes fluid flow problems. We first present the FCBI procedure and specifically the flow-condition-based interpolations used, and then give demonstrative solutions to illustrate the capacity of the scheme. These solutions include the use of regular and irregular grids, with coarse and fine meshes, and for low and higher Reynolds number flows of well-chosen test problems. We concentrate in this paper on the formulation of the proposed discretization scheme, based on the objectives given above, and the detailed solutions of some test problems. Although we consider in this study only steady-state conditions, the proposed method can also be applied to time-dependent problems as is the FCBI method based 
on quadrilateral grids [8]. Of course, a full evaluation of the scheme should also include a study of its numerical efficiency when the scheme is embedded in a complete CFD computer code. Such study should then comprise the accuracy of the scheme, and the number of iterations used and the numerical effort per iteration, when compared to using other CFD discretization methods, in the solution of complex and perhaps even industrial problems. However, such comprehensive evaluation is beyond the scope of this paper.

\section{A NEW FCBI METHOD FOR THE SOLUTION OF NAVIER-STOKES EQUATIONS}

In this section, we present an FCBI method using triangular grids for the analysis of incompressible fluid flows. We first give the mathematical model considered and then present the procedure based on the MINI element used [9].

\subsection{Governing equations and finite element formulation}

We consider a two-dimensional steady-state fluid flow problem governed by the incompressible Navier-Stokes equations. We assume that the problem is well-posed in the Hilbert spaces $V$ and $P$. The non-dimensional governing equations in conservative form are:

Find the velocity $\mathbf{v}(\mathbf{x}) \in V$ and pressure $p(\mathbf{x}) \in P$ such that

$$
\begin{aligned}
\nabla \cdot \mathbf{v}=0, & \mathbf{x} \in \Omega \\
\nabla \cdot(\mathbf{v v}-\tau)=\mathbf{0}, & \mathbf{x} \in \Omega
\end{aligned}
$$

subject to the boundary conditions

$$
\begin{array}{rr}
\mathbf{v}=\mathbf{v}^{s}, & \mathbf{x} \in \bar{S}_{v} \\
\tau \cdot \mathbf{n} & =\mathbf{f}^{s}, \quad \mathbf{x} \in S_{f}
\end{array}
$$

where $\Omega \in \Re^{2}$ is a domain with the boundary $S=\bar{S}_{v} \cup S_{f}\left(S_{v} \cap S_{f}=\emptyset\right)$, $\tau$ is the stress tensor defined as

$$
\tau=\tau(\mathbf{v}, p)=-p \mathbf{I}+\frac{1}{R e}\left\{\nabla \mathbf{v}+(\nabla \mathbf{v})^{\mathrm{T}}\right\}
$$

with the identity tensor $\mathbf{I}$ and the Reynolds number $R e, \mathbf{v}^{s}$ is the prescribed velocity on the boundary $\bar{S}_{v}, \mathbf{f}^{s}$ is the prescribed traction on the boundary $S_{f}$, and $\mathbf{n}$ is the unit normal vector to the boundary.

For the finite element solution, we use a Petrov-Galerkin variational formulation with subspaces $U_{h}, V_{h}$ and $W_{h}$ of $V$, and $P_{h}$ and $Q_{h}$ of $P$ of the problem in Equations (1)-(4). The formulation for the numerical solution is:

Find $\mathbf{u} \in U_{h}, \mathbf{v} \in V_{h}$ and $p \in P_{h}$ such that for all $w \in W_{h}$ and $q \in Q_{h}$ :

$$
\int_{\Omega} w \nabla \cdot(\mathbf{u v}-\tau(\mathbf{u}, p)) \mathrm{d} \Omega=\mathbf{0}
$$




$$
\int_{\Omega} q \nabla \cdot \mathbf{u} \mathrm{d} \Omega=0
$$

The trial functions in $U_{h}$ and $P_{h}$ are the usual functions of finite element interpolations for velocity and pressure, respectively. These are selected to satisfy the inf-sup condition of incompressible analysis [9]. An important point is that the trial functions in $V_{h}$ are different from the functions in $U_{h}$ and are defined using the flow conditions in order to stabilize the advection term. The weight functions in the spaces $W_{h}$ and $Q_{h}$ are step functions, which enforce the local conservation of momentum and mass, respectively.

\subsection{Using the MINI element}

To establish an FCBI scheme for triangular grids that can be used to solve problems with complex geometries, we develop a new method that possesses the basic ingredients mentioned above, i.e. interpolations to satisfy the inf-sup condition, the use of the flow conditions in the trial functions and step functions as weight functions. The procedure using the MINI element is detailed in this section.

Figure 1 shows a MINI element in which the velocity is defined at four nodes, the local node numbers 1-4, while the pressure is defined at three nodes, the local node numbers $1-3$, in order to satisfy the inf-sup condition. With the use of step weight functions around nodes, the control volumes in the spaces $W_{h}$ and $Q_{h}$ are considered as shown in Figures 2(a) and (b), respectively. The flux is calculated with the interpolated values at the centre of the sides of the control volumes. The velocity $\mathbf{u}$ and the pressure $p$ are obtained with the trial functions in

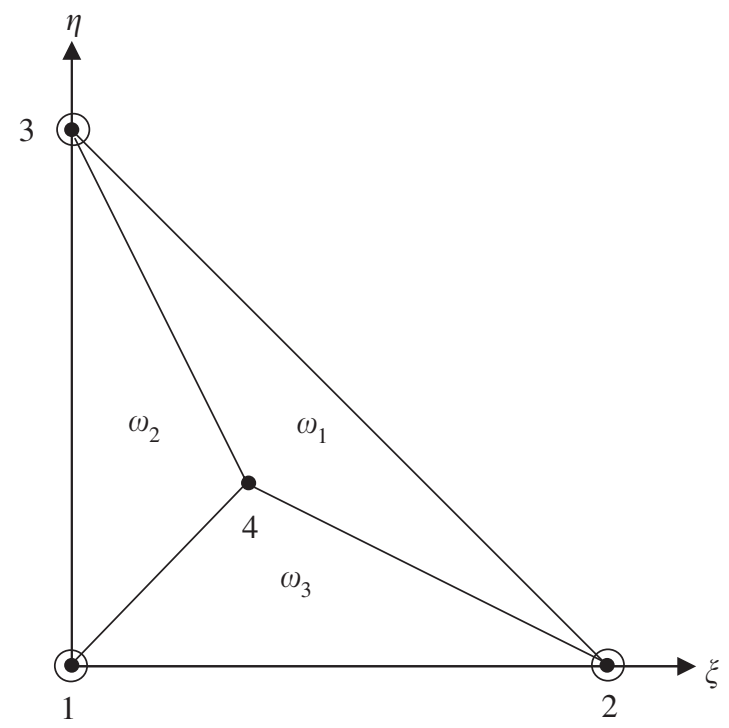

Figure 1. A MINI element. 

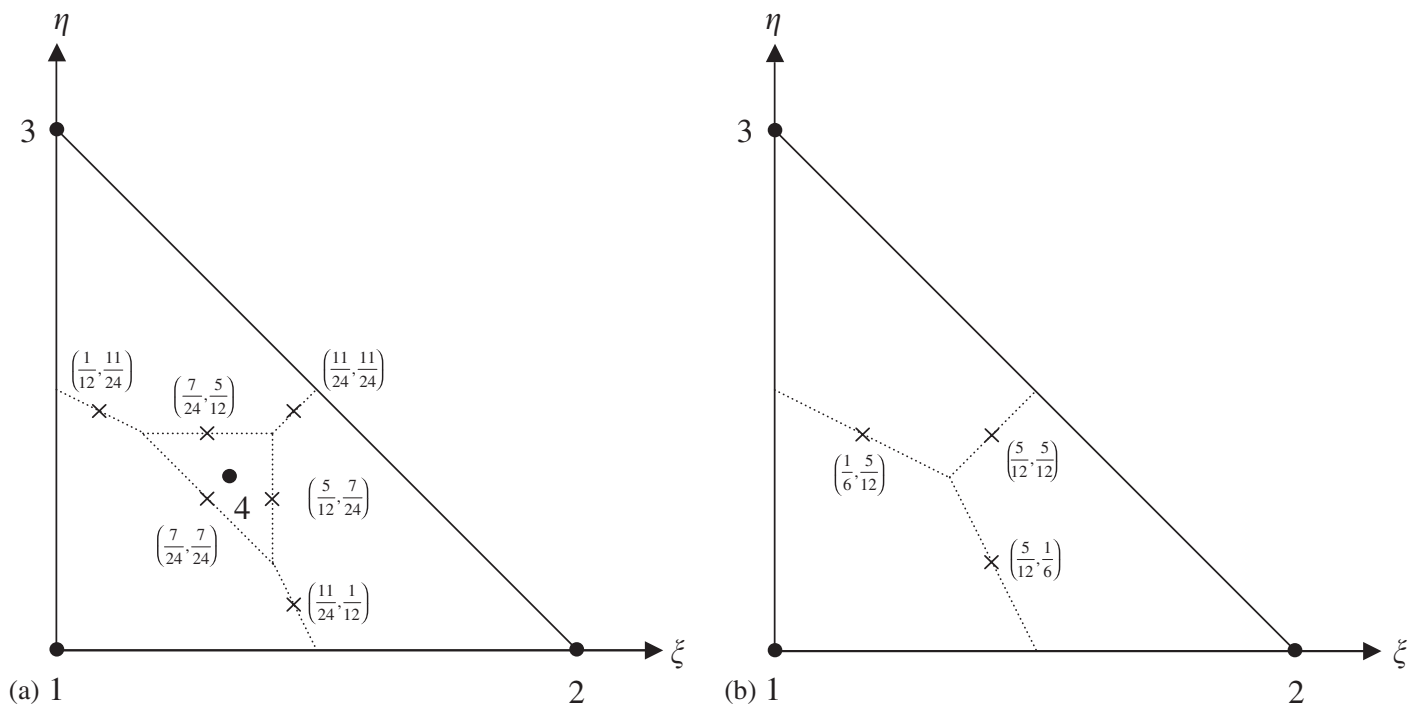

Figure 2. Control volumes and flux calculation points: (a) segment in the space $W_{h}$; and (b) segment in the space $Q_{h}$.

Table I. Trial functions in $U_{h}$ and nodal co-ordinates.

\begin{tabular}{lccc}
\hline Trial function & $\mathrm{i}$ & $\xi$ & $\eta$ \\
\hline$h_{1}^{u}=1-\xi-\eta-\phi_{e} / 3$ & 1 & 0 & 0 \\
$h_{2}^{u}=\xi-\phi_{e} / 3$ & 2 & 1 & 0 \\
$h_{3}^{u}=\eta-\phi_{e} / 3$ & 3 & 0 & 1 \\
$h_{4}^{u}=\phi_{e}$ & 4 & $1 / 3$ & $1 / 3$ \\
\hline
\end{tabular}

Table II. Trial functions in $P_{h}$ and nodal co-ordinates.

\begin{tabular}{llll}
\hline Trial function & i & $\xi$ & $\eta$ \\
\hline$h_{1}^{p}=1-\xi-\eta$ & 1 & 0 & 0 \\
$h_{2}^{p}=\xi$ & 2 & 1 & 0 \\
$h_{3}^{p}=\eta$ & 3 & 0 & 1 \\
\hline
\end{tabular}

$U_{h}$ and $P_{h}$ given in Tables I and II:

$$
\begin{aligned}
& \mathbf{u}=h_{i}^{u} \mathbf{v}_{i} \\
& p=h_{i}^{p} p_{i}
\end{aligned}
$$




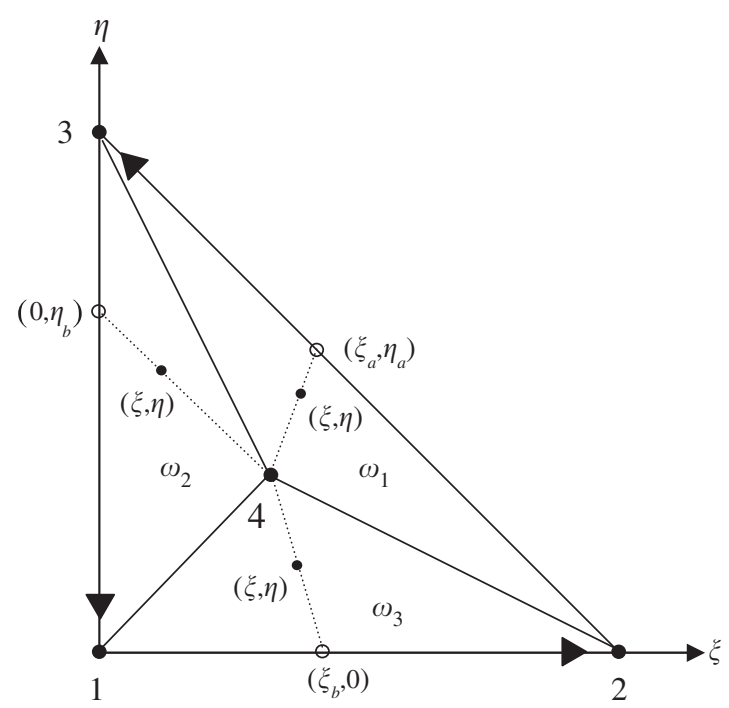

Figure 3. Values used in the construction of the trial functions in $V_{h}$.

where $\mathbf{v}_{i}$ and $p_{i}$ are the nodal velocity and pressure variables, respectively. The bubble function $\phi_{e}$ is defined as

$$
\phi_{e}= \begin{cases}3(1-\xi-\eta) & \text { in } \omega_{1} \\ 3 \xi & \text { in } \omega_{2} \\ 3 \eta & \text { in } \omega_{3}\end{cases}
$$

in the three domains shown in Figure 1.

In order to reach a stable solution scheme, the velocity $\mathbf{v}$ in the advection term is interpolated using the flow conditions, see Figure 3. As in the original FCBI method, the flow conditions are evaluated on the sides of the element with an analytical solution of the one-dimensional advection-diffusion equation. However, we consider here different interpolation functions for the velocity components $v_{\|}$and $v_{\perp}$ that are measured, respectively, parallel and perpendicular to each side of the three domains $\omega_{1}, \omega_{2}$ and $\omega_{3}$. The flow-condition-based interpolation is applied to the parallel component, while linear interpolation is employed to the perpendicular component. This improves the accuracy of the solution (see Remark 1 below). The trial functions for the parallel component $h_{i \|}^{v}$ in $V_{h}$ are given in Table III, and the functions for both components are attached to the same nodal velocities used in Equation (8) as follows:

$$
\begin{gathered}
v_{\|}=h_{i \|}^{v} v_{i \|}=h_{i}^{v} v_{i \|} \\
v_{\perp}=h_{i \perp}^{v} v_{i \perp}=h_{i}^{u} v_{i \perp}
\end{gathered}
$$


FLOW-CONDITION-BASED INTERPOLATION FINITE ELEMENT PROCEDURE

Table III. Trial functions in $V_{h}$ for the velocity component parallel to the element sides.

\begin{tabular}{lccc}
\hline & $\omega_{1}$ & $\omega_{2}$ & $\omega_{3}$ \\
\hline$h_{1 \|}^{v}$ & 0 & $(1-3 \xi) x^{31}$ & $(1-3 \eta)\left(1-x^{12}\right)$ \\
$h_{2 \|}^{v}$ & $(3 \xi+3 \eta-2)\left(1-x^{23}\right)$ & 0 & $(1-3 \eta) x^{12}$ \\
$h_{3 \|}^{v}$ & $(3 \xi+3 \eta-2) x^{23}$ & $(1-3 \xi)\left(1-x^{31}\right)$ & 0 \\
$h_{4 \|}^{v}$ & $3(1-\xi-\eta)$ & $3 \xi$ & $3 \eta$ \\
\hline
\end{tabular}

with

$$
\begin{aligned}
x^{12} & =\frac{\mathrm{e}^{R e_{12}^{e} \xi_{b}}-1}{\mathrm{e}^{R e_{12}^{e}}-1}, \quad x^{23}=\frac{\mathrm{e}^{R e_{23}^{e} \eta_{a}}-1}{\mathrm{e}^{R e_{23}^{e}}-1}, \quad x^{31}=\frac{\mathrm{e}^{R e_{31}^{e}\left(1-\eta_{b}\right)}-1}{\mathrm{e}^{R e_{31}^{e}}-1} \\
\xi_{b} & =\frac{-\xi+\eta}{3 \eta-1}, \quad \eta_{a}=\frac{\xi+2 \eta-1}{3 \xi+3 \eta-2}, \quad \eta_{b}=\frac{\xi-\eta}{3 \xi-1} \\
R e_{12}^{e} & =\operatorname{Re} \overline{\mathbf{v}}_{12} \cdot \Delta \mathbf{x}_{12}, \quad \operatorname{Re} e_{23}^{e}=\operatorname{Re} \overline{\mathbf{v}}_{23} \cdot \Delta \mathbf{x}_{23}, \quad \operatorname{Re} e_{31}^{e}=\operatorname{Re} \overline{\mathbf{v}}_{31} \cdot \Delta \mathbf{x}_{31} \\
\overline{\mathbf{v}}_{12} & =\frac{1}{2}\left(\mathbf{v}_{1}+\mathbf{v}_{2}\right), \quad \overline{\mathbf{v}}_{23}=\frac{1}{2}\left(\mathbf{v}_{2}+\mathbf{v}_{3}\right), \quad \overline{\mathbf{v}}_{31}=\frac{1}{2}\left(\mathbf{v}_{3}+\mathbf{v}_{1}\right) \\
\Delta \mathbf{x}_{12} & =\mathbf{x}_{2}-\mathbf{x}_{1}, \quad \Delta \mathbf{x}_{23}=\mathbf{x}_{3}-\mathbf{x}_{2}, \quad \Delta \mathbf{x}_{31}=\mathbf{x}_{1}-\mathbf{x}_{3}
\end{aligned}
$$

where $R e_{12}^{e}, R e_{23}^{e}$ and $R e_{31}^{e}$ are the element Reynolds numbers on the sides 1-2, 2-3 and 3-1, respectively, and $\mathbf{x}_{i}=\left(x_{i}, y_{i}\right)$ for $i=1,2,3$ are the nodal co-ordinates. The trial functions for the perpendicular component $h_{i \perp}^{v}$ in $V_{h}$ are obtained by replacing $x^{23}, x^{31}$ and $x^{12}$ with $\eta_{a}, 1-\eta_{b}$ and $\xi_{b}$, respectively, and they are the same as the functions used in Equation (8). Note that the bubble function $\phi_{e}$ is not changed as shown in Table III.

The proposed trial functions have the following properties:

- Stability is obtained through the introduction of the flow-condition interpolation.

- No artificial parameters are employed.

- Compatibility between adjacent domains (i.e. $\omega_{i}$ and $\omega_{j}$ for $i, j=1,2,3$ ) is satisfied.

- The requirement $\Sigma h_{i}^{v}=1$ is satisfied.

- An interpolated value at a specific point does not depend on the node numbering.

- The functions are always positive.

- The functions are invariant to a rotation of the Cartesian co-ordinate system.

- As the element Reynolds numbers become small, the trial functions in $V_{h}$ approach the trial functions in $U_{h}$. (This is proved by substituting $x^{12} \cong \xi_{b}, x^{23} \cong \eta_{a}, x^{31} \cong 1-\eta_{b}$ into the functions listed in Table III.)

Although the flow conditions are taken counterclockwise in Table III, which corresponds to the direction in Figure 3, it is of course also possible to consider the flow conditions clockwise due 
to the following relation:

$$
x^{21}=1-x^{12}, \quad x^{32}=1-x^{23}, \quad x^{13}=1-x^{31}
$$

Hence, geometrically, the trial functions in $V_{h}$ correspond to a linear interpolation between the values at the centroid and the point on the side whose position is determined by the natural co-ordinate $(\xi, \eta)$ as shown in Figure 3 . The points $\left(\xi_{a}, \eta_{a}\right),\left(0, \eta_{b}\right)$ and $\left(\xi_{b}, 0\right)$ correspond to the intersections of the sides and the lines that connect the centroid and the interpolating positions, and the values at these points are calculated according to the analytical solution of the advectiondiffusion equation. This can be described by the following equations:

$$
\begin{aligned}
v_{\|}^{1}= & \frac{\left\{\left(\xi-\frac{1}{3}\right)^{2}+\left(\eta-\frac{1}{3}\right)^{2}\right\}^{1 / 2}}{\left\{\left(\xi_{a}-\frac{1}{3}\right)^{2}+\left(\eta_{a}-\frac{1}{3}\right)^{2}\right\}^{1 / 2}}\left\{\left(1-x^{23}\right) v_{2 \|}+x^{23} v_{3 \|}\right\} \\
& +\frac{\left\{\left(\xi_{a}-\xi\right)^{2}+\left(\eta_{a}-\eta\right)^{2}\right\}^{1 / 2}}{\left\{\left(\xi_{a}-\frac{1}{3}\right)^{2}+\left(\eta_{a}-\frac{1}{3}\right)^{2}\right\}^{1 / 2}} v_{4 \|} \\
= & (3 \xi+3 \eta-2)\left(1-x^{23}\right) v_{2 \|}+(3 \xi+3 \eta-2) x^{23} v_{3 \|}+3(1-\xi-\eta) v_{4 \|} \\
v_{\|}^{2}= & \frac{\left\{\left(\xi-\frac{1}{3}\right)^{2}+\left(\eta-\frac{1}{3}\right)^{2}\right\}^{1 / 2}}{\left\{\frac{1}{9}+\left(\eta_{b}-\frac{1}{3}\right)^{2}\right\}^{1 / 2}}\left\{x^{31} v_{1 \|}+\left(1-x^{31}\right) v_{3 \|}\right\} \\
& +\frac{\left\{\xi^{2}+\left(\eta_{b}-\eta\right)^{2}\right\}^{1 / 2}}{\left\{\frac{1}{9}+\left(\eta_{b}-\frac{1}{3}\right)^{2}\right\}^{1 / 2}} v_{4 \|} \\
= & (1-3 \xi) x^{31} v_{1 \|}+(1-3 \xi)\left(1-x^{31}\right) v_{3 \|}+3 \xi v_{4 \|} \\
v_{\|}^{3}= & \frac{\left\{\left(\xi-\frac{1}{3}\right)^{2}+\left(\eta-\frac{1}{3}\right)^{2}\right\}^{1 / 2}}{\left\{\left(\xi_{b}-\frac{1}{3}\right)^{2}+\frac{1}{9}\right\}^{1 / 2}}\left\{\left(1-x^{12}\right) v_{1 \|}+x^{12} v_{2 \|}\right\} \\
& +\frac{\left\{\left(\xi_{b}-\xi\right)^{2}+\eta^{2}\right\}^{1 / 2}}{\left\{\left(\xi_{b}-\frac{1}{3}\right)^{2}+\frac{1}{9}\right\}^{1 / 2}} v_{4 \|} \\
= & (1-3 \eta)\left(1-x^{12}\right) v_{1 \|}+(1-3 \eta) x^{12} v_{2 \|}+3 \eta v_{4 \|}
\end{aligned}
$$

where $v_{\|}^{1}, v_{\|}^{2}$ and $v_{\|}^{3}$ are the interpolated parallel components of the velocities $\mathbf{v}^{1}, \mathbf{v}^{2}$ and $\mathbf{v}^{3}$ at $(\xi, \eta)$ in $\omega_{1}, \omega_{2}$ and $\omega_{3}$, respectively, which are described using the unit vectors as follows:

$$
v_{\|}^{1}=\mathbf{v}^{1} \cdot \mathbf{e}_{\|}^{23}, \quad v_{\|}^{2}=\mathbf{v}^{2} \cdot \mathbf{e}_{\|}^{31}, \quad v_{\|}^{3}=\mathbf{v}^{3} \cdot \mathbf{e}_{\|}^{12}
$$

with

$$
\mathbf{e}_{\|}^{23}=\frac{\Delta \mathbf{x}_{23}}{\left\|\Delta \mathbf{x}_{23}\right\|}, \quad \mathbf{e}_{\|}^{31}=\frac{\Delta \mathbf{x}_{31}}{\left\|\Delta \mathbf{x}_{31}\right\|}, \quad \mathbf{e}_{\|}^{12}=\frac{\Delta \mathbf{x}_{12}}{\left\|\Delta \mathbf{x}_{12}\right\|}
$$




\section{Remark 1}

In the original FCBI technique proposed for quadrilateral elements [6], the flow-condition-based interpolations were constructed using the flow conditions along opposing element sides, with an interpolation over the element. The two sets of opposing element sides were used. However, in the formulation of triangular elements, the flow conditions along each of three element sides need to be considered in an equal manner to reach an isotropic element. This isotropy and in addition a rational scheme for good predictive capability are achieved by decomposing the velocity vector into the parallel and perpendicular components to each element side and using different trial functions for the components (see Section 3.2 for results obtained when compared to using the same flow-condition-based interpolations for parallel and perpendicular velocity components). Notice that the element Reynolds number defined in Equation (12) can be rewritten in the following form:

$$
\begin{aligned}
\operatorname{Re}_{12}^{e} & =\operatorname{Re} \overline{\mathbf{v}}_{12} \cdot \Delta \mathbf{x}_{12} \\
& =\operatorname{Re} \bar{v}_{\| 12}\left\|\Delta \mathbf{x}_{12}\right\| \\
& =\operatorname{Re} \frac{v_{1 \|}+v_{2 \|}}{2}\left\|\Delta \mathbf{x}_{12}\right\|
\end{aligned}
$$

Since the parallel components of velocities to the side 1-2, which are defined at the nodes 1 and 2, are used, the advected velocity should also be parallel to the side. Hence, in the scheme proposed in this paper, the perpendicular component of velocity is interpolated linearly as the element Reynolds number is considered to be infinitesimal due to $\Delta \mathbf{x}_{12} \cdot \mathbf{e}_{12 \perp}=0$ where $\mathbf{e}_{12 \perp}$ is the unit vector perpendicular to the side 1-2.

\section{Remark 2}

In the Cartesian co-ordinate systems, the velocity in the domain $\omega_{1}$ and its components are written as follows:

$$
\begin{aligned}
\mathbf{v}^{1}= & v_{\|}^{1} \mathbf{e}_{\|}^{23}+v_{\perp}^{1} \mathbf{e}_{\perp}^{23} \\
= & v_{x}^{1} \mathbf{e}_{x}+v_{y}^{1} \mathbf{e}_{y} \\
v_{x}^{1}= & v_{\|}^{1}\left(e_{\|}^{23}\right)_{x}+v_{\perp}^{1}\left(e_{\perp}^{23}\right)_{x} \\
= & (3 \xi+3 \eta-2)\left[\left(1-x^{23}\right)\left\{\left(e_{\|}^{23}\right)_{x}\right\}^{2}+\left(1-\eta_{a}\right)\left\{\left(e_{\perp}^{23}\right)_{x}\right\}^{2}\right] v_{2 x} \\
& +(3 \xi+3 \eta-2)\left[\left(1-x^{23}\right)\left(e_{\|}^{23}\right)_{x}\left(e_{\|}^{23}\right)_{y}+\left(1-\eta_{a}\right)\left(e_{\perp}^{23}\right)_{x}\left(e_{\perp}^{23}\right)_{y}\right] v_{2 y} \\
& +(3 \xi+3 \eta-2)\left[x^{23}\left\{\left(e_{\|}^{23}\right)_{x}\right\}^{2}+\eta_{a}\left\{\left(e_{\perp}^{23}\right)_{x}\right\}^{2}\right] v_{3 x} \\
& +(3 \xi+3 \eta-2)\left[x^{23}\left(e_{\|}^{23}\right)_{x}\left(e_{\|}^{23}\right)_{y}+\eta_{a}\left(e_{\perp}^{23}\right)_{x}\left(e_{\perp}^{23}\right)_{y}\right] v_{3 y} \\
& +3(1-\xi-\eta) v_{4 x} \\
= & \alpha_{1 x} v_{2 x}+\alpha_{2 x} v_{2 y}+\alpha_{3 x} v_{3 x}+\alpha_{4 x} v_{3 y}+\alpha_{5 x} v_{4 x}
\end{aligned}
$$




$$
\begin{aligned}
v_{y}^{1}= & v_{\|}^{1}\left(e_{\|}^{23}\right)_{y}+v_{\perp}^{1}\left(e_{\perp}^{23}\right)_{y} \\
= & (3 \xi+3 \eta-2)\left[\left(1-x^{23}\right)\left(e_{\|}^{23}\right)_{x}\left(e_{\|}^{23}\right)_{y}+\left(1-\eta_{a}\right)\left(e_{\perp}^{23}\right)_{x}\left(e_{\perp}^{23}\right)_{y}\right] v_{2 x} \\
& +(3 \xi+3 \eta-2)\left[\left(1-x^{23}\right)\left\{\left(e_{\|}^{23}\right)_{y}\right\}^{2}+\left(1-\eta_{a}\right)\left\{\left(e_{\perp}^{23}\right)_{y}\right\}^{2}\right] v_{2 y} \\
& +(3 \xi+3 \eta-2)\left[x^{23}\left(e_{\|}^{23}\right)_{x}\left(e_{\|}^{23}\right)_{y}+\eta_{a}\left(e_{\perp}^{23}\right)_{x}\left(e_{\perp}^{23}\right)_{y}\right] v_{3 x} \\
& +(3 \xi+3 \eta-2)\left[x^{23}\left\{\left(e_{\|}^{23}\right)_{y}\right\}^{2}+\eta_{a}\left\{\left(e_{\perp}^{23}\right)_{y}\right\}^{2}\right] v_{3 y} \\
& +3(1-\xi-\eta) v_{4 y} \\
= & \alpha_{1 y} v_{2 x}+\alpha_{2 y} v_{2 y}+\alpha_{3 y} v_{3 x}+\alpha_{4 y} v_{3 y}+\alpha_{5 y} v_{4 y}
\end{aligned}
$$

with

$$
\begin{aligned}
\mathbf{e}_{\perp}^{23} & =\mathbf{e}_{x} \times \mathbf{e}_{y} \times \mathbf{e}_{\|}^{23} \\
\left(e_{\|}^{23}\right)_{x} & =\mathbf{e}_{\|}^{23} \cdot \mathbf{e}_{x}, \quad\left(e_{\|}^{23}\right)_{y}=\mathbf{e}_{\|}^{23} \cdot \mathbf{e}_{y} \\
\left(e_{\perp}^{23}\right)_{x} & =\mathbf{e}_{\perp}^{23} \cdot \mathbf{e}_{x}, \quad\left(e_{\perp}^{23}\right)_{y}=\mathbf{e}_{\perp}^{23} \cdot \mathbf{e}_{y}
\end{aligned}
$$

where $\mathbf{e}_{x}$ and $\mathbf{e}_{y}$ are the unit vectors in the $x$ and $y$ directions, respectively. The velocity components $v_{x}^{2}, v_{y}^{2}, v_{x}^{3}$ and $v_{y}^{3}$ can also be obtained in a similar way. Note that the requirement $\Sigma h_{i}^{v}=1$, i.e. $\Sigma \alpha_{i x}=\Sigma \alpha_{i y}=1$ in Equations (21) and (22), is still satisfied since $\left(e_{\|}^{23}\right)_{x}=\left(e_{\perp}^{23}\right)_{y}$ and $\left(e_{\|}^{23}\right)_{y}=-\left(e_{\perp}^{23}\right)_{x}$. In addition, it is readily confirmed from Equations (21) and (22) that $v_{x}^{1}$ and $v_{y}^{1}$ are independent of the directions of $\mathbf{e}_{\|}^{23}$ and $\mathbf{e}_{\perp}^{23}$ as long as these unit vectors are, respectively, parallel and perpendicular to the side 2-3. The same holds for the other components.

\section{NUMERICAL EXAMPLES}

In this section, the performance of the new FCBI method is evaluated using some test problems. First, we apply the proposed scheme to the solution of an advection-diffusion problem for which the exact analytical solution exists. Then we solve two Navier-Stokes flow problems: a lid-driven flow in a square cavity and in a triangular cavity. The full Newton-Raphson method is used to solve the nonlinear equations with the convergence criteria $\max \left(R_{v}\right) \leqslant 10^{-6}$ and $\max \left(R_{p}\right) \leqslant 10^{-6}$ where $R_{v}=\|\Delta \mathbf{v}\| /\|\mathbf{v}\|, R_{p}=|\Delta p| /|p|$. To reach the solutions for higher Reynolds numbers, we use the converged solution of the lower Reynolds number case as initial condition.

\subsection{Solution of an advection-diffusion temperature problem between parallel plates}

We include the solution of this problem in order to compare our calculated results with an analytical solution. Figure 4 shows the analytical model of the temperature problem considered with the boundary conditions and the mesh of $30 \times 30 \times 2$ elements used in this study (see also Reference [7]). When a unit velocity is prescribed in the $x$ direction over the whole domain, 


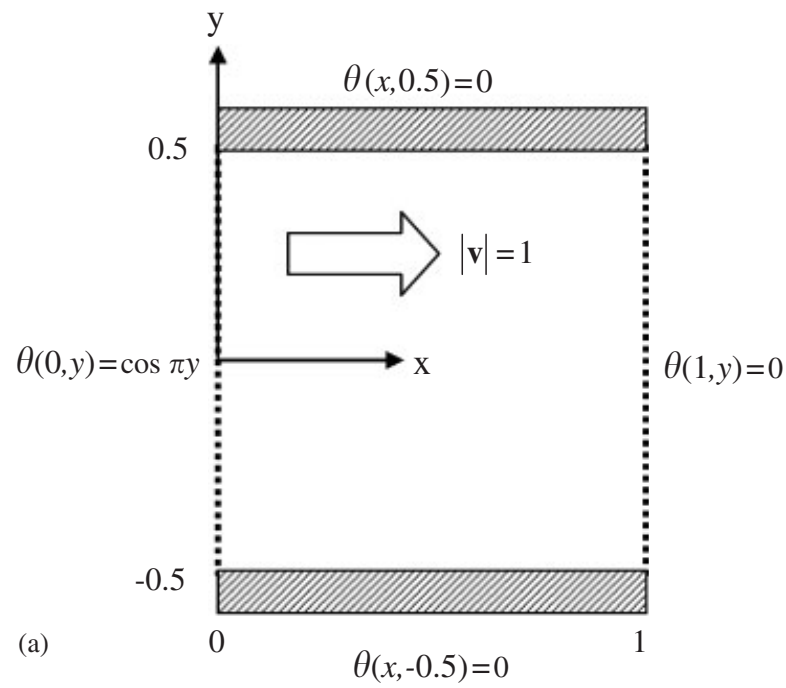

(b)

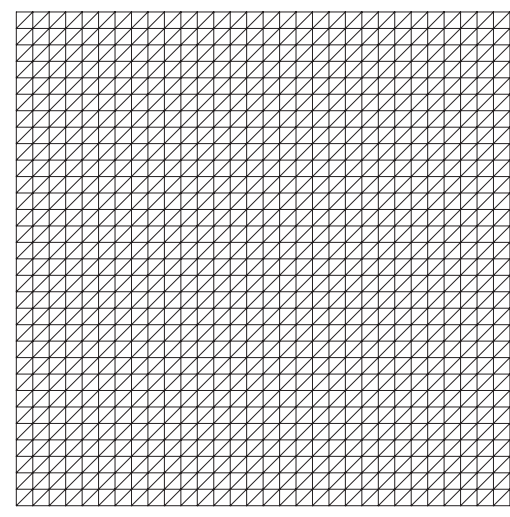

Figure 4. The flow problem between parallel plates and the mesh used: (a) problem definition; and (b) regular triangular mesh.

the exact steady-state solution for the temperature $\theta$ is

$$
\theta(x, y)=\frac{\cos \pi y}{\mathrm{e}^{a}-\mathrm{e}^{b}}\left(\mathrm{e}^{a+b x}-\mathrm{e}^{b+a x}\right)
$$

with

$$
a=\frac{1}{2}\left(P e+\sqrt{P e^{2}+4 \pi^{2}}\right), \quad b=\frac{1}{2}\left(P e-\sqrt{P e^{2}+4 \pi^{2}}\right)
$$

where $P e$ is the Péclet number.

Figures 5 and 6 show the comparison of temperature values on the centre line and on vertical lines through the channel for $P e=10,100$ and 1000 . Although the calculated values deviate slightly from the exact data near the right boundary for $P e=100$ and 1000 , good agreement with the exact solutions can be seen in all cases.

\subsection{Solution of driven flow in a square cavity}

The capability of the scheme for Navier-Stokes flow problems is next assessed by solving the lid-driven flow problem in a square cavity. This problem is widely used as a benchmark to evaluate developed numerical schemes. We compare our numerical results with the solutions of Ghia et al. [11] which are regarded as accurate.

Figures $7(a)$ and (b) show the geometry of the square cavity with the co-ordinate system and the nomenclature used for the centres and representative lengths of the vortices, respectively. The no-slip boundary condition is imposed on the left, lower and right boundaries, while a unit velocity is prescribed on the upper boundary including the corners. In addition, zero pressure is prescribed at the lower left corner. Three types of regular meshes and an irregular mesh are used in the analysis for the fluid flow up to the Reynolds number 10000 . Figures 8(a)-(c) show the 

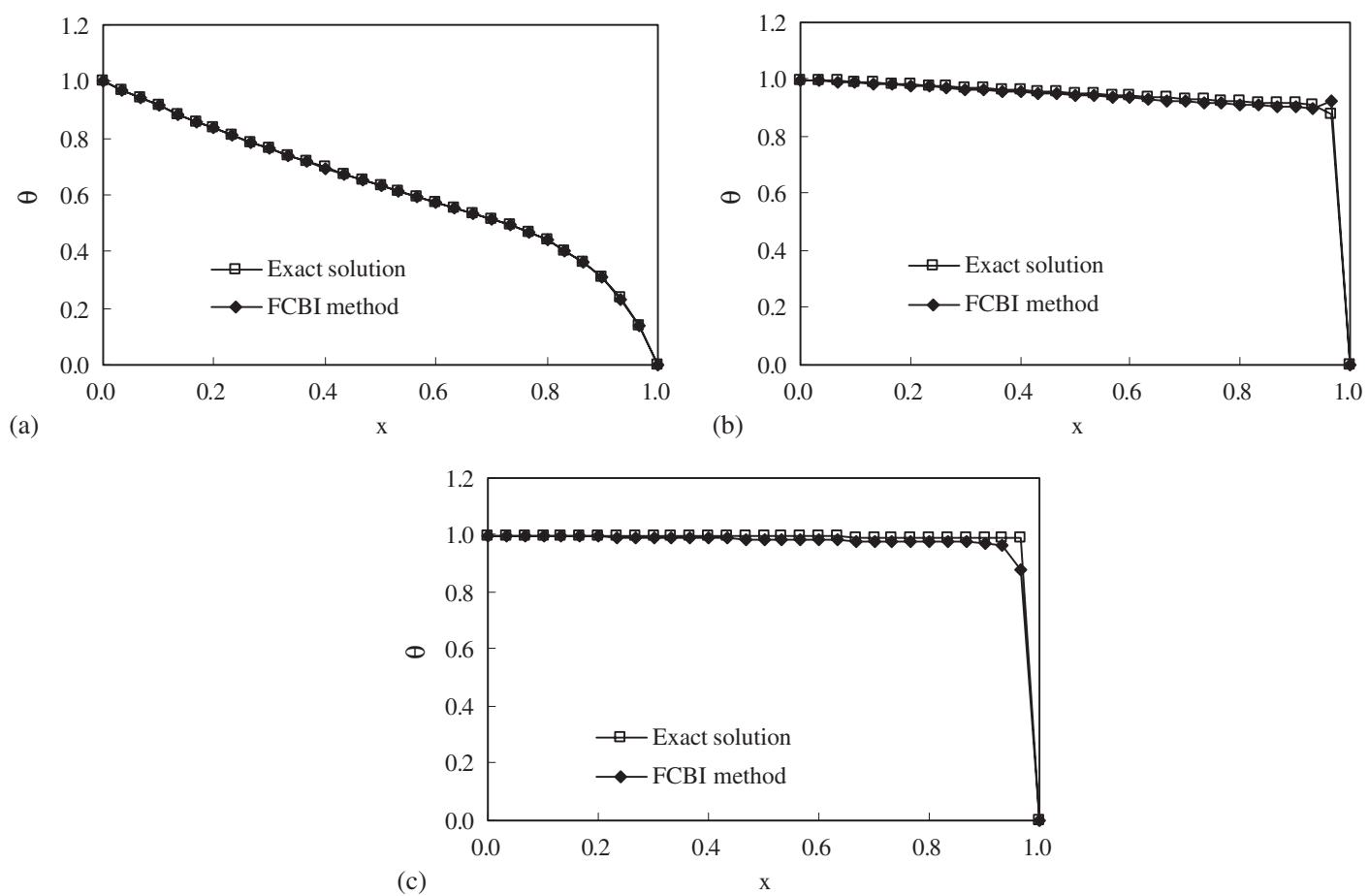

Figure 5. Comparison of temperature values on the centre line: (a) $P e=10$; (b) $P e=100$; and (c) $P e=1000$.

regular meshes including $40 \times 40 \times 2$ elements, which are named Meshes $1-3$, respectively, and Figure 8(d) shows the irregular mesh named Mesh 4 that consists of $20 \times 20 \times 2$ elements. In Meshes 1-3, the grid points are distributed finer near the boundary according to the following equations:

$$
\begin{aligned}
& x(i)=\frac{\mathrm{e}^{(2 \gamma / N)(i-1)}-1}{2\left(\mathrm{e}^{\gamma}-1\right)} L \quad\left(1 \leqslant i \leqslant \frac{N}{2}+1\right) \\
& x(i)=\left\{1-\frac{\mathrm{e}^{(2 \gamma / N)(N+1-i)}-1}{2\left(\mathrm{e}^{\gamma}-1\right)}\right\} L \quad\left(\frac{N}{2}+1 \leqslant i \leqslant N+1\right)
\end{aligned}
$$

where $N$ is the number of elements on a side, $L$ is the length of the side, $i$ is the node number and $\gamma$ represents the parameter for unequal division. The value of $\gamma$ is fixed at 2 for the three meshes.

First, the fluid flow for $R e=10000$ is calculated using Mesh 1, and the obtained velocity profiles along the centre lines are shown in Figure 9. For the display of the results, we use the $r, s$ co-ordinate systems along the centre lines $(-1 \leqslant r, s \leqslant 1)$ defined in Figure 7(a). As an experiment, we also show the solution obtained if the same flow-condition-based interpolations are used for the parallel and perpendicular components of velocity on the element sides. Although for this high Reynolds number a finer mesh is necessary to reach agreement with the result of Ghia 


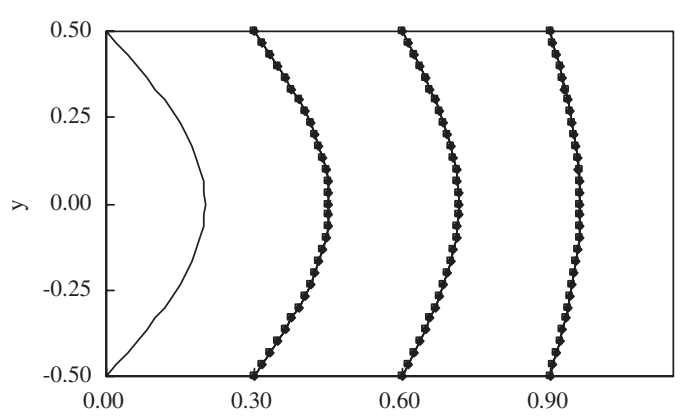

(a)

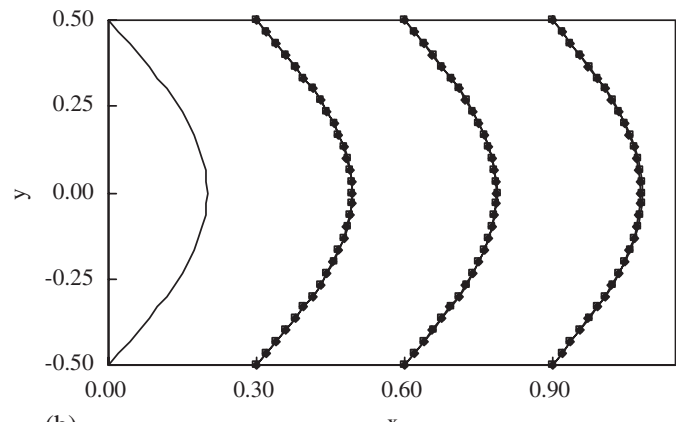

(b)

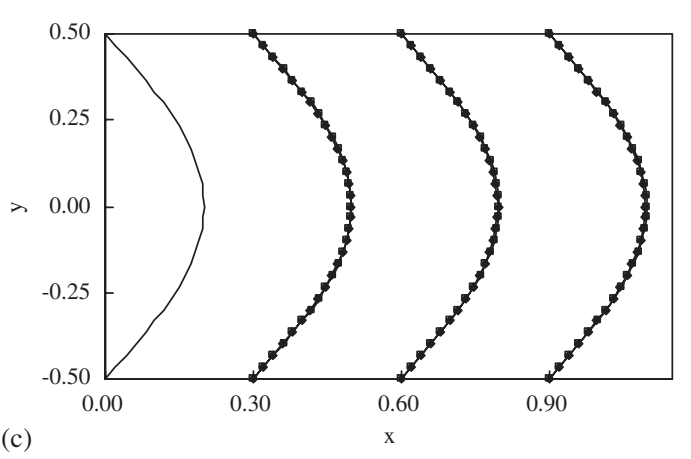

(c)

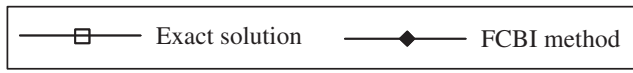

Figure 6. Comparison of temperature profiles on vertical lines:

(a) $P e=10$; (b) $P e=100$; and (c) $P e=1000$.

et al. [11] (see below), the proposed approach of using different interpolations for the parallel and perpendicular components of velocity is more effective, see also Remark 1 .

The dependence of solutions on the meshes used is evaluated in Figure 10, for Meshes 1-3. The obtained results with the three meshes for $R e=1000$ are close to each other in velocity profiles along the centre lines and agree reasonably well with those of Ghia et al. [11].

The results obtained using the unstructured grid, Mesh 4, are given in Figure 11 for the case $R e=1000$. Reasonable results are obtained using this coarse and distorted mesh, which indicates the robustness of the FCBI scheme.

Figure 12 shows the comparison of our results with those of Ghia et al. [11] for the cases $R e=5000$ and 10000 . In order to obtain more accurate results for these high Reynolds number flows, we use a mesh based on the element distribution of Mesh 1 but with the number of elements increased to $160 \times 160 \times 2$. The velocity profiles along the centre lines are in good agreement with those reported by Ghia et al. [11].

Figures 13 and 14 show the streamline patterns and vorticity contours, respectively, obtained with the $160 \times 160 \times 2$ mesh for $R e=1000,5000$ and 10000 . The vorticity is defined as $\omega=\left[\left(\partial v_{y} / \partial x\right)-\left(\partial v_{x} / \partial y\right)\right]$, and the contours are drawn at intervals of $\Delta \omega=1.0$ for a range of $-10.0 \leqslant \omega \leqslant 3.0$. As the Reynolds number increases, the vorticity in the primary vortex becomes 


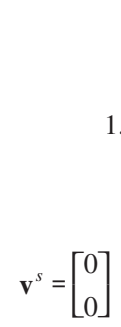

(a) 0.0

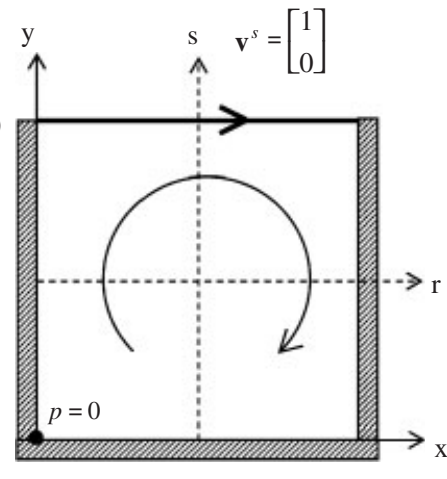

1.0

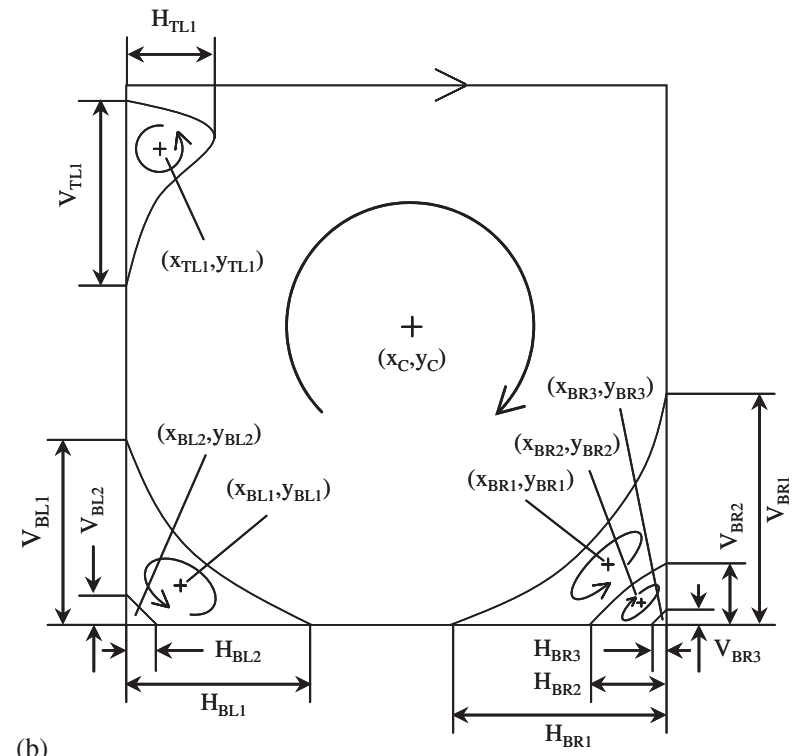

(b)

Figure 7. Square-cavity flow problem: (a) problem definition $(-1 \leqslant r, s \leqslant 1)$; and (b) nomenclature (taken from Reference [11]).

almost constant due to the near-linearity of the velocity profiles, see Figure 12, whereas the vorticity changes significantly near the boundary.

A comparison of some characteristic values with the results of Ghia et al. [11] for $R e=10000$ is listed in Table IV using the nomenclature in Figure 7(b). The vortex centres and the representative lengths corresponding to the velocity profile in Figure 12(b) are in good agreement with those reported by Ghia et al. [11] in which a $257 \times 257$ mesh is used. Note that the smallest secondary vortex in the bottom right corner is captured with fewer elements per side than those in the mesh used by Ghia et al.

\subsection{Solution of driven flow in a triangular cavity}

As a second fluid flow example, we consider the driven flow in an equilateral triangular cavity. For this problem solution, triangular grids are quite natural to use. Although triangularcavity flows have been studied by some researchers $[12,13]$, the flows considered were of rather small Reynolds numbers. Here we solve small and large Reynolds number flows; the maximum Reynolds number is 10 times larger than that reported by Ribbens et al. [12] in the same analytical model.

Figures 15(a) and (b) show the geometry of the triangular cavity with the co-ordinate system and the nomenclature for the vortices. As in the square-cavity flow problem, the no-slip boundary condition is imposed on the left and right boundaries, while a unit velocity is prescribed on the top boundary. At the bottom corner, the pressure is fixed at zero. The calculation is conducted using two types of regular meshes named Mesh 1 and Mesh 2, for which the element patterns are, respectively, shown in Figures 16(a) and (b), and consist of $l(l+1)$ and $l^{2} / 2$ elements, where 


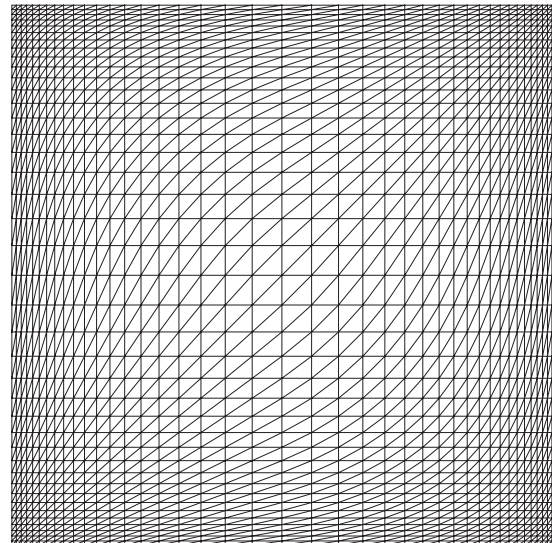

(a)

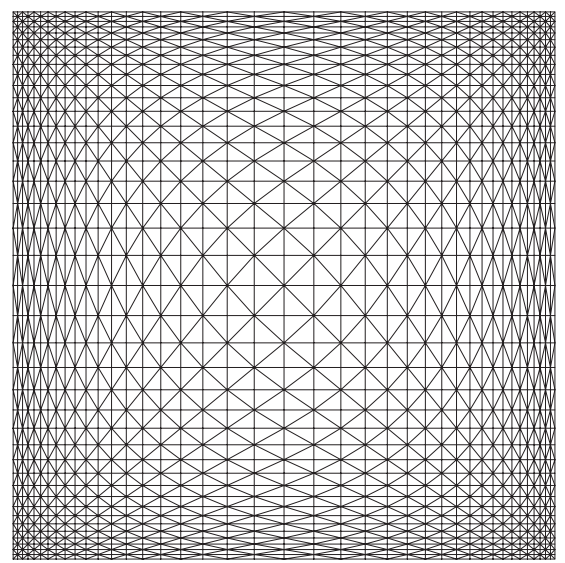

(c)

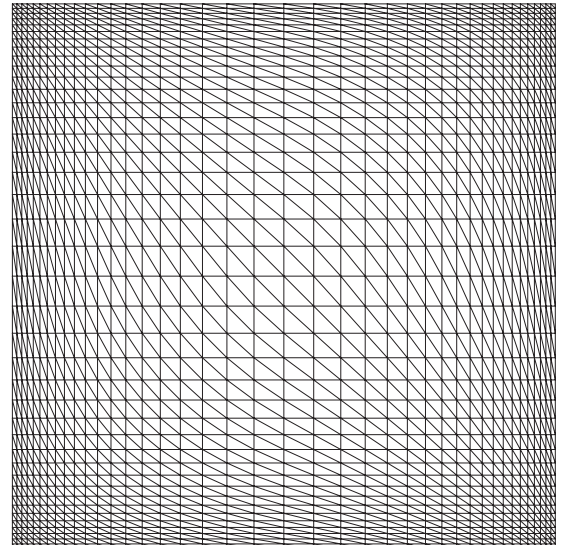

(b)

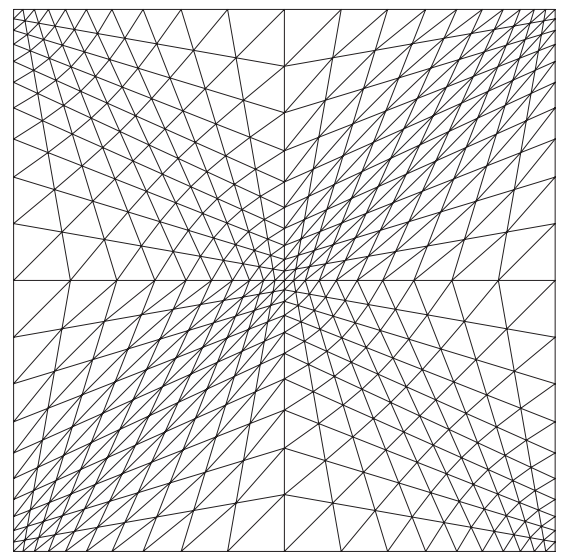

(d)

Figure 8. (a) Mesh 1; (b) Mesh 2; (c) Mesh 3; and (d) Mesh 4.

$l$ is the number of elements along the top wall. In this analysis, 44310 elements $(l=210)$ for Mesh 1 and 45000 elements $(l=300)$ for Mesh 2 are used up to the Reynolds number 5000 .

Figure 17 shows the velocity profiles in the $x$ direction along the centre line and the $y$ direction along the horizontal line at $y=-1$ in the cavity obtained with Meshes 1 and 2 for the cases $R e=100,500$ and 5000. For the display of the results, we use in this figure the $r, s$ co-ordinate systems $(-1 \leqslant r, s \leqslant 1)$ defined in Figure $15(\mathrm{a})$. As in the square-cavity flow problem, the nearly linear variation of the velocity and the kinks near $y=0$ on the centre line and $x=\frac{2}{\sqrt{3}}$ on the horizontal line are observed for the case $R e=5000$. Since the solutions in Meshes 1 and 2 are almost the same for these Reynolds numbers, the obtained results are not sensitive to the meshes used.

As demonstrated in Section 3.2, the present scheme provides not only stable results even with distorted grids but also accurate results, with the accuracy of course dependent on the fineness of the mesh. Hence, we deem it useful to give more details of our results and we list some calculated 


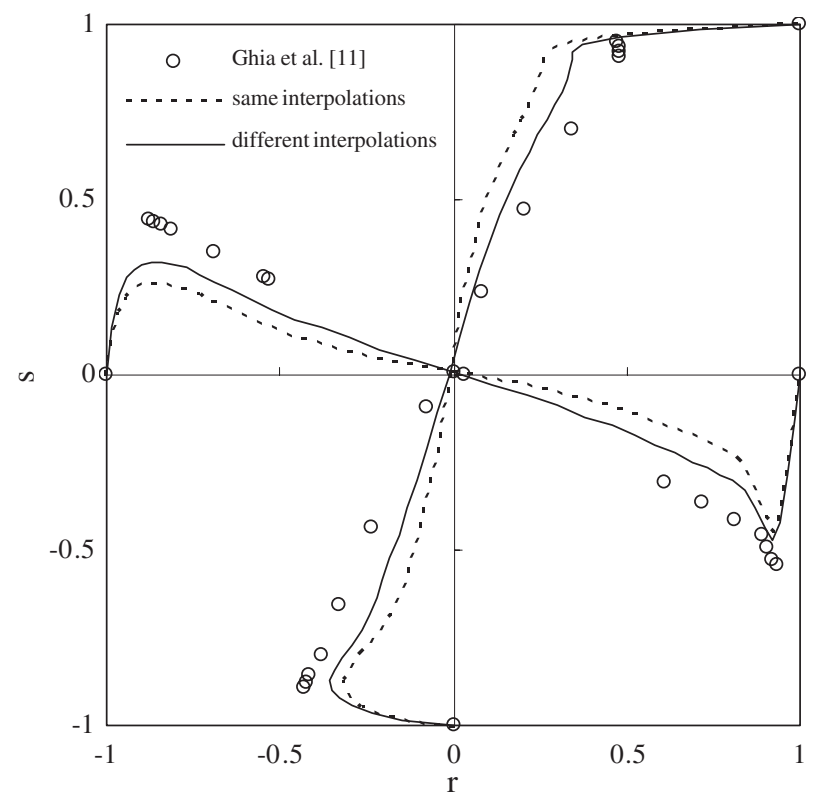

Figure 9. Comparison of vertical and horizontal velocity profiles along the centre lines obtained with two different types of interpolations for $R e=10000$.

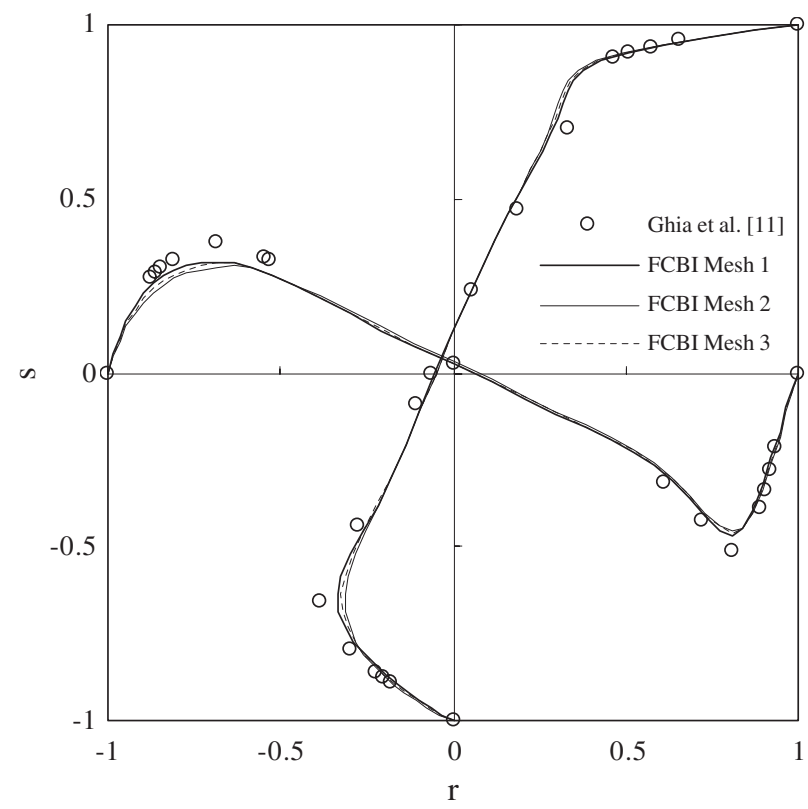

Figure 10. Comparison of vertical and horizontal velocity profiles along the centre lines obtained with three different meshes for $R e=1000$. 


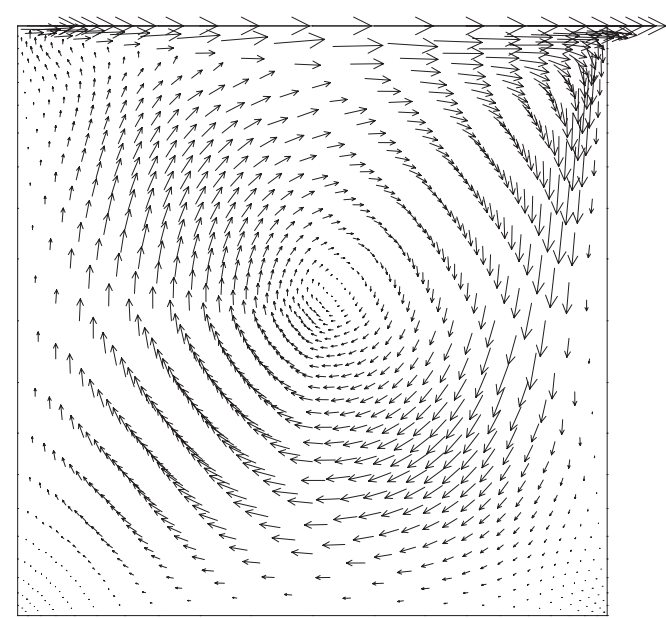

(a)

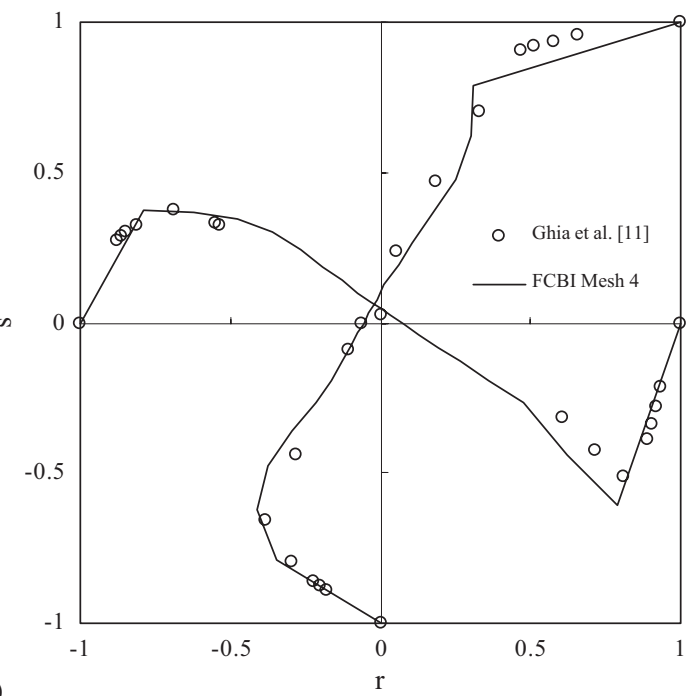

(b)

Figure 11. Solutions in Mesh 4 for $R e=1000$ : (a) velocity distribution; and (b) comparison of vertical and horizontal velocity profiles along the centre lines.
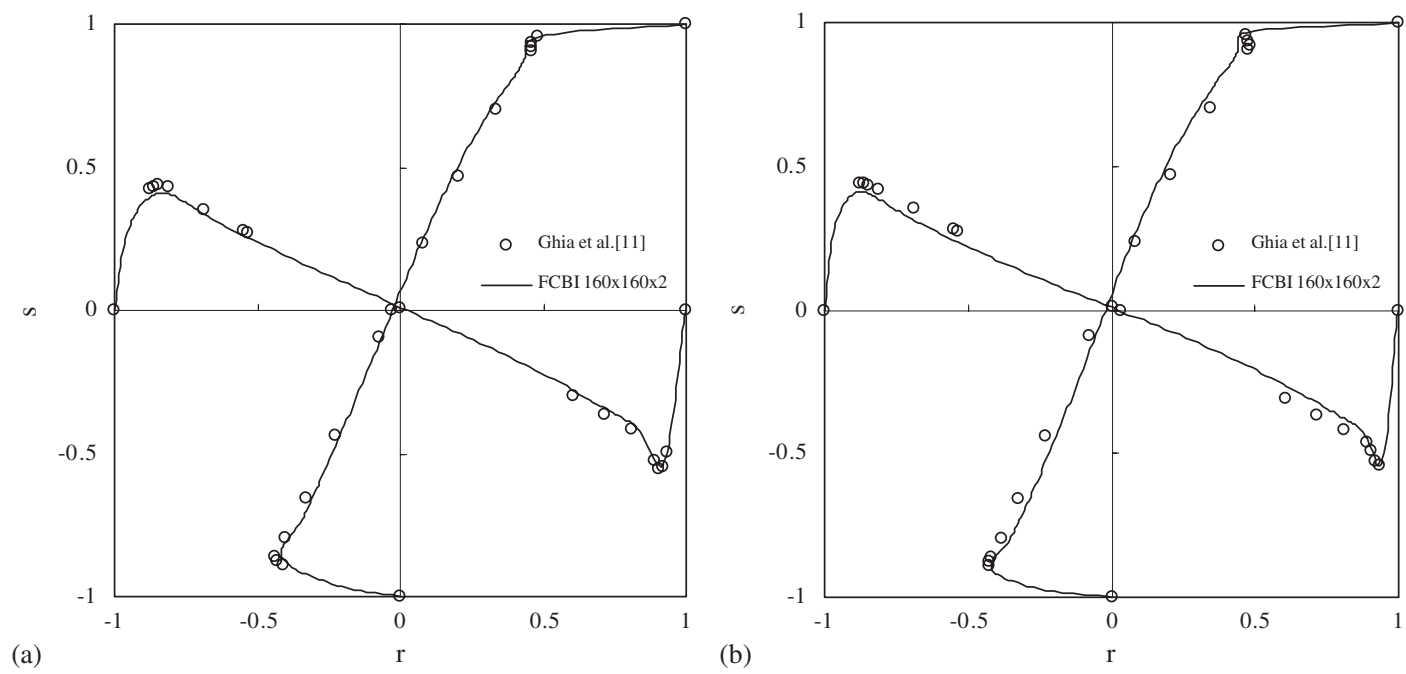

Figure 12. Comparison of vertical and horizontal velocity profiles along the centre lines: (a) solution for $R e=5000$; and (b) solution for $R e=10000$.

values in Tables V and VI, in which local maxima and minima are underlined. Noting that the results for Meshes 1 and 2 are virtually the same, we show in Tables V and VI, respectively, the $x$-velocity along the centre line obtained from Mesh 1 and the $y$-velocity along the horizontal 


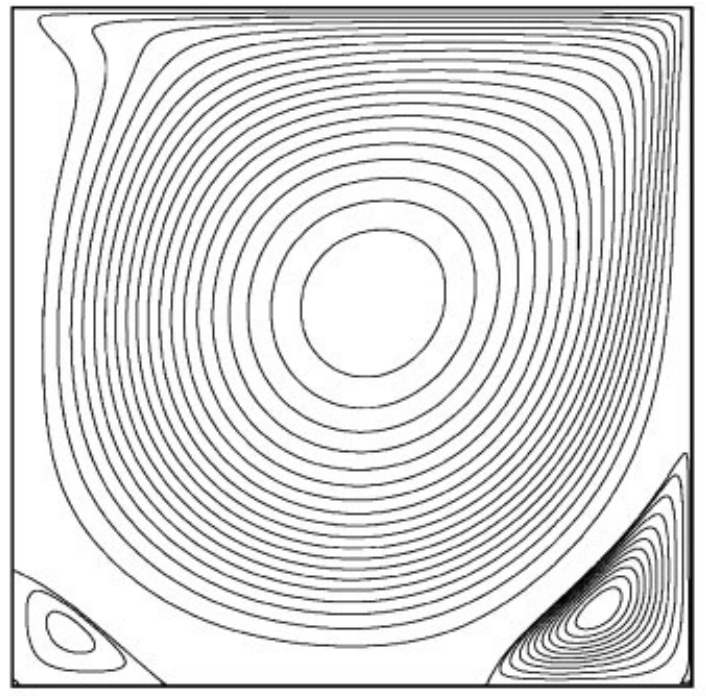

(a)

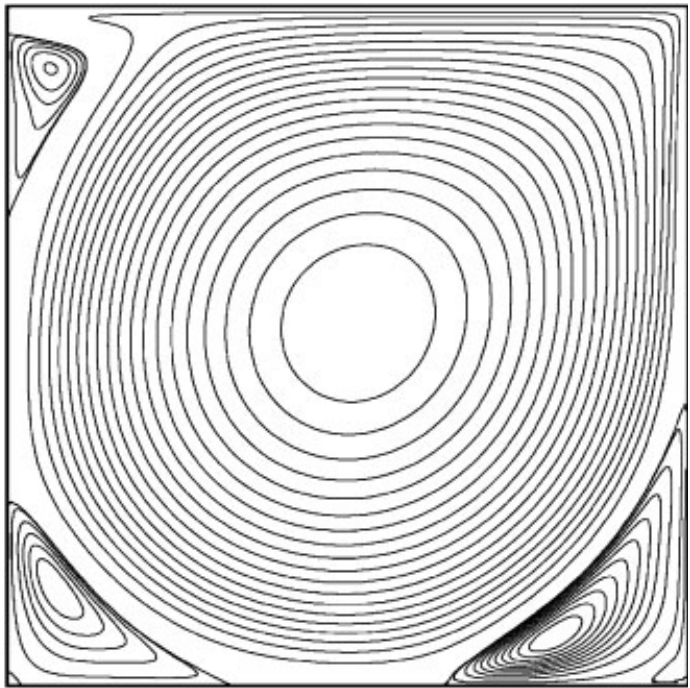

(b)

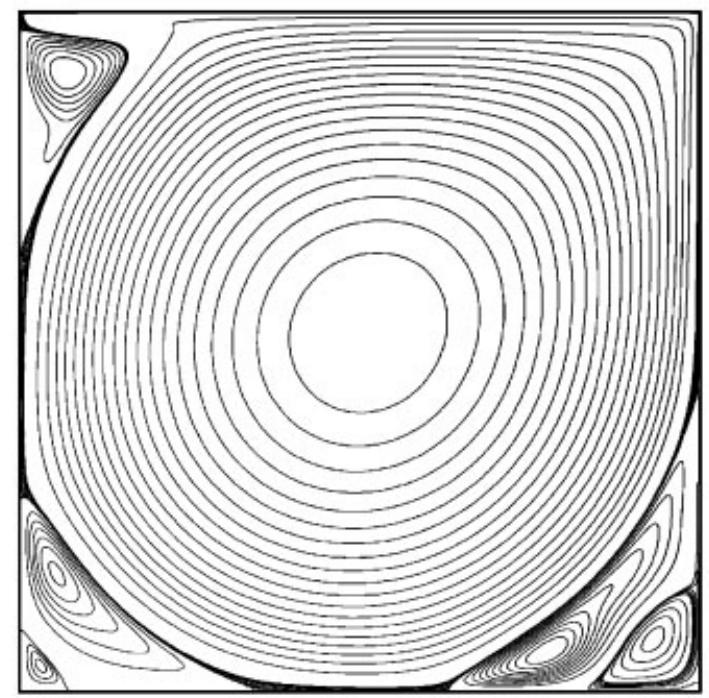

(c)

Figure 13. Streamline patterns: (a) solution for $R e=1000$; (b) solution for $R e=5000$; and (c) solution for $R e=10000$.

line at $y=-1$ from Mesh 2. Showing the results this way, we have more data points than if the results were employed from one mesh only.

Figures 18 and 19 show the streamline patterns and vorticity contours, respectively, obtained with Mesh 1 for $R e=100,500$ and 5000. In Figure 18, we see that some vortices appear around the primary vortex, and their number increases as the Reynolds number increases. The vorticity 


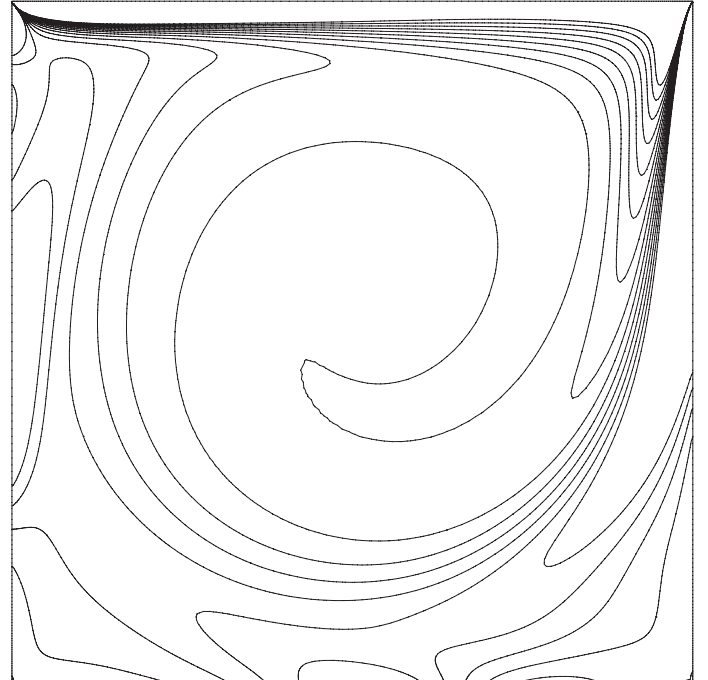

(a)

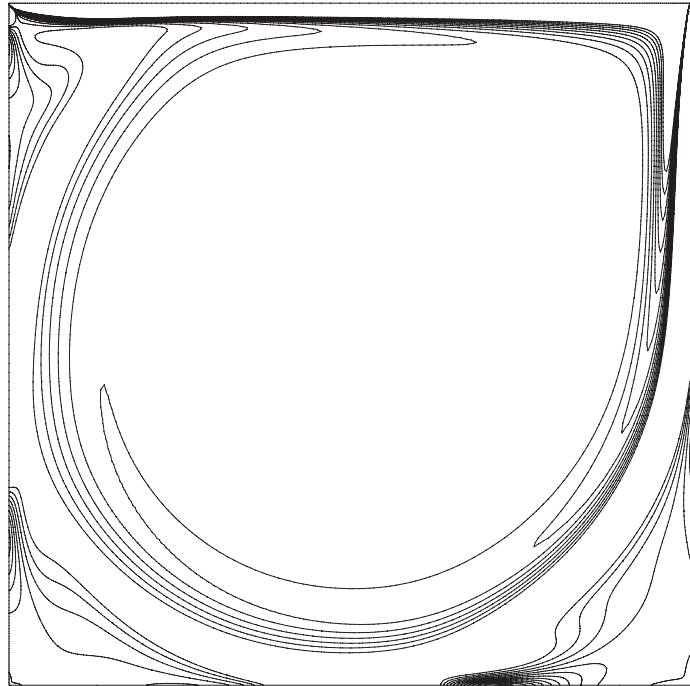

(b)

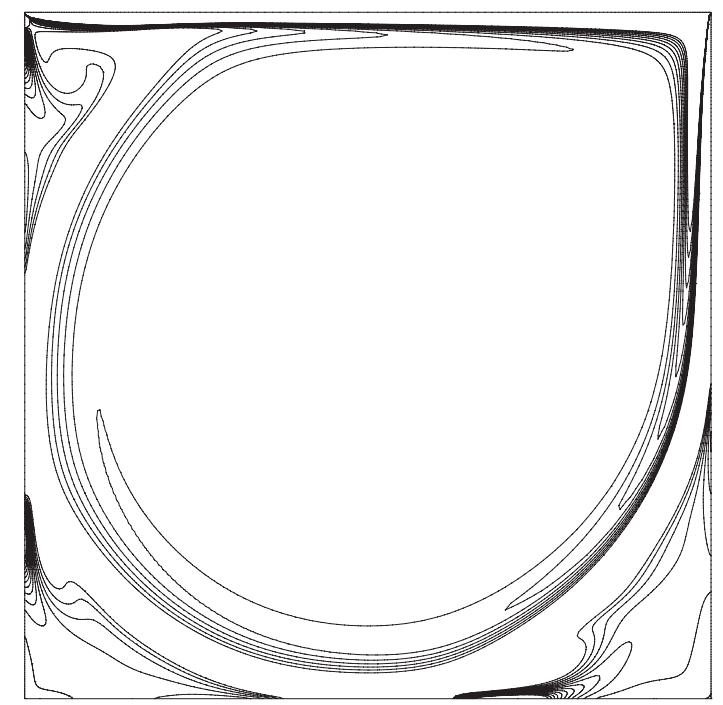

(c)

Figure 14. Vorticity contours: (a) solution for $R e=1000$; (b) solution for $R e=5000$; and (c) solution for $R e=10000$.

contours are drawn at intervals of $\Delta \omega=0.5$ for a range of $-5.0 \leqslant \omega \leqslant 5.0$. As in the squarecavity flow, the vorticity in the primary vortex is approximately constant for $R e=5000$, while the gradient of vorticity becomes large between the vortices and near the geometric boundary.

The locations of the centres of the vortices according to the nomenclature in Figure 15(b) are listed in Table VII. Among them, the locations in the primary vortex can be compared 
Table IV. Comparison of characteristic values for $R e=10000$.

\begin{tabular}{lcc}
\hline Parameter & FCBI & Ghia et al. $[11]$ \\
\hline$\left(x_{\mathrm{C}}, y_{\mathrm{C}}\right)$ & $(0.5127,0.5291)$ & $(0.5117,0.5333)$ \\
$\left(x_{\mathrm{TL} 1}, y_{\mathrm{TL} 1}\right)$ & $(0.0682,0.9116)$ & $(0.0703,0.9141)$ \\
$\left(x_{\mathrm{BL} 1}, y_{\mathrm{BL} 1}\right)$ & $(0.0530,0.1732)$ & $(0.0586,0.1641)$ \\
$\left(x_{\mathrm{BL} 2}, y_{\mathrm{BL} 2}\right)$ & $(0.0305,0.0372)$ & $(0.0156,0.0195)$ \\
$\left(x_{\mathrm{BR} 1}, y_{\mathrm{BR} 1}\right)$ & $(0.7777,0.0581)$ & $(0.7656,0.0586)$ \\
$\left(x_{\mathrm{BR} 2}, y_{\mathrm{BR} 2}\right)$ & $(0.9326,0.0763)$ & $(0.9336,0.0625)$ \\
$\left(x_{\mathrm{BR} 3}, y_{\mathrm{BR} 3}\right)$ & $(0.9958,0.0043)$ & $(0.9961,0.0039)$ \\
$\mathrm{H}_{\mathrm{TL} 1}$ & 0.1537 & 0.1589 \\
$\mathrm{~V}_{\mathrm{TL} 1}$ & 0.3241 & 0.3203 \\
$\mathrm{H}_{\mathrm{BL} 1}$ & 0.3455 & 0.3438 \\
$\mathrm{~V}_{\mathrm{BL} 1}$ & 0.2857 & 0.2891 \\
$\mathrm{H}_{\mathrm{BL} 2}$ & 0.0685 & 0.0352 \\
$\mathrm{~V}_{\mathrm{BL} 2}$ & 0.0896 & 0.0441 \\
$\mathrm{H}_{\mathrm{BR} 1}$ & 0.3730 & 0.3906 \\
$\mathrm{~V}_{\mathrm{BR} 1}$ & 0.4455 & 0.4492 \\
$\mathrm{H}_{\mathrm{BR} 2}$ & 0.1699 & 0.1706 \\
$\mathrm{~V}_{\mathrm{BR} 2}$ & 0.1576 & 0.1367 \\
$\mathrm{H}_{\mathrm{BR} 3}$ & 0.0089 & 0.0039 \\
$\mathrm{~V}_{\mathrm{BR} 3}$ & 0.0092 & 0.0039 \\
\hline
\end{tabular}

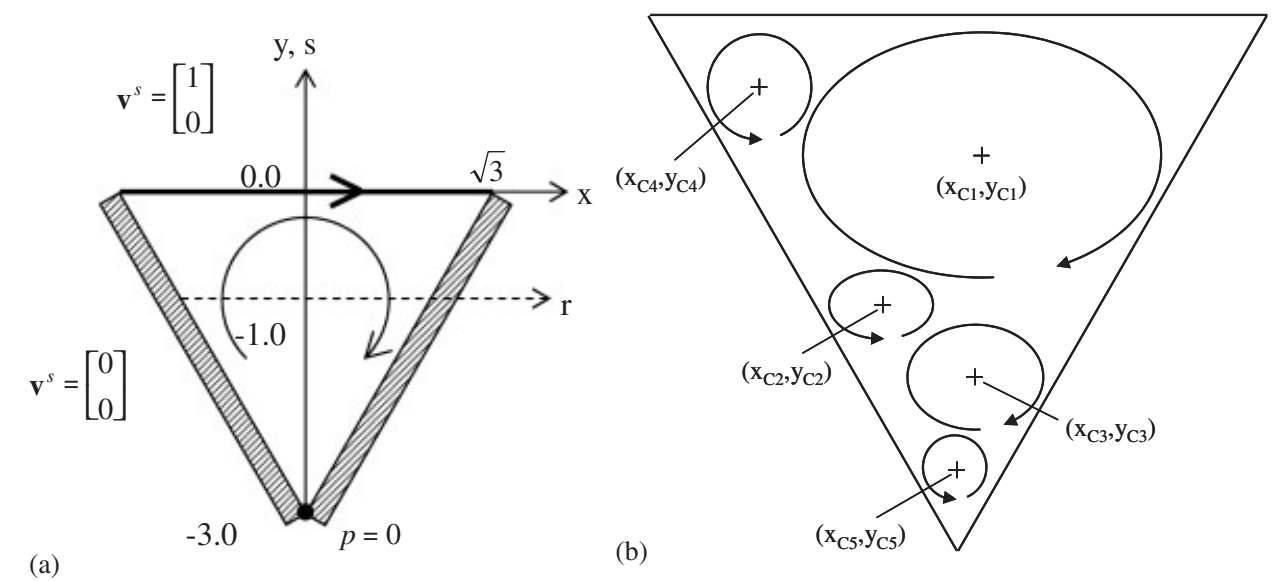

Figure 15. Triangular-cavity flow problem: (a) problem definition $(-1 \leqslant r, s \leqslant 1)$; and (b) nomenclature.

with the results of Ribbens et al. [12]. Although the present results agree well with those of Ribbens et al. [12] for the cases $R e=100$ and 200, we obtained somewhat different data when $R e=500$. To study our results further we plot the calculated centre position of the primary vortex as a function of the Reynolds number in Figure 20(a). This position changes smoothly according to the development of the flow field; hence our results for $R e=500$ are deemed accurate. 

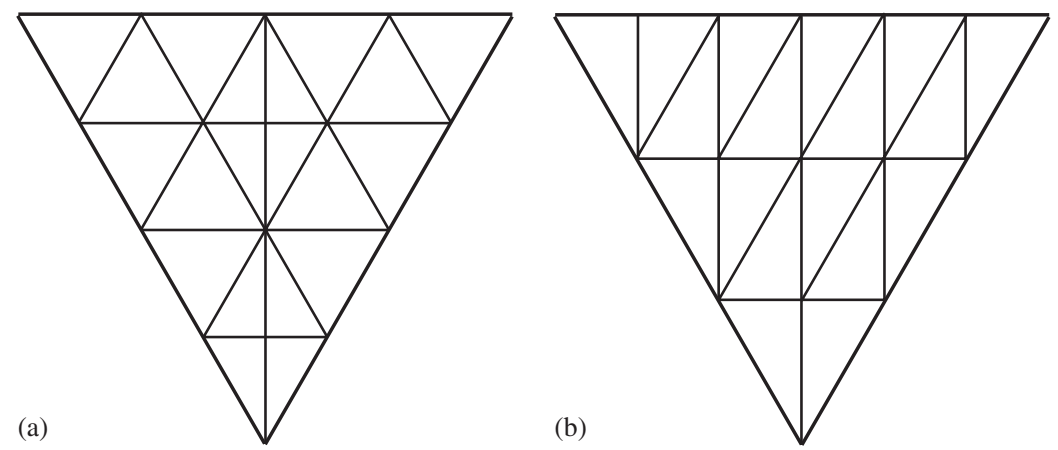

Figure 16. (a) Mesh 1; and (b) Mesh 2.
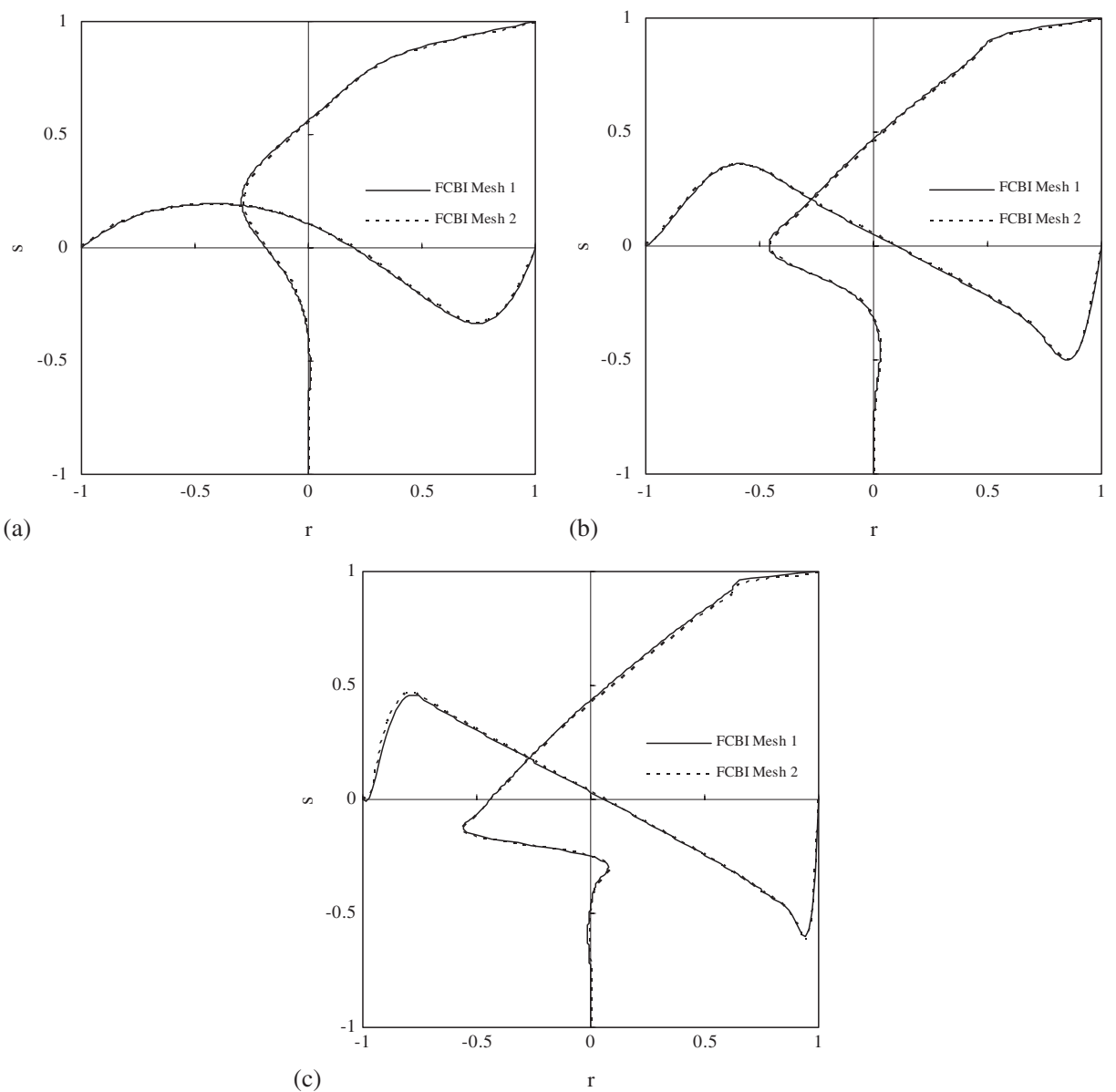

Figure 17. Vertical and horizontal velocity profiles along the centre line $(x=0)$ and the horizontal line $(y=-1)$ obtained with two different meshes: (a) solution for $\operatorname{Re}=100$; (b) solution for $R e=500$; and (c) solution for $R e=5000$. 
Table V. Velocity values in the $x$ direction along the centre line $(x=0)$ in Mesh 1.

\begin{tabular}{rrrrrrrrr}
\hline & & \multicolumn{7}{c}{$R e$} \\
\cline { 3 - 8 } Grid pt. no. & \multicolumn{1}{c}{$y$} & \multicolumn{1}{c}{100} & \multicolumn{1}{c}{ 200 } & \multicolumn{1}{c}{500} & \multicolumn{1}{c}{1000} & 2000 & \multicolumn{1}{c}{3500} & \multicolumn{1}{c}{5000} \\
\hline 211 & 0.0000 & 1.00000 & 1.00000 & 1.00000 & 1.00000 & 1.00000 & 1.00000 & 1.00000 \\
207 & -0.0571 & 0.81766 & 0.78999 & 0.73838 & 0.69877 & 0.66698 & 0.65422 & 0.65324 \\
206 & -0.0714 & 0.77171 & 0.73771 & 0.67965 & 0.64323 & 0.62494 & 0.62693 & 0.63440 \\
205 & -0.0857 & 0.72676 & 0.68813 & 0.62977 & 0.60396 & 0.60359 & 0.61767 & 0.62950 \\
204 & -0.1000 & 0.68320 & 0.64201 & 0.58927 & 0.57817 & 0.59334 & 0.61342 & 0.62599 \\
180 & -0.4429 & 0.16395 & 0.23983 & 0.28014 & 0.29736 & 0.31156 & 0.32091 & 0.32664 \\
150 & -0.8714 & -0.16653 & -0.10758 & -0.05682 & -0.03738 & -0.02639 & -0.02100 & -0.01853 \\
128 & -1.1857 & -0.29476 & -0.33718 & -0.26938 & -0.25167 & -0.24414 & -0.24211 & -0.24228 \\
119 & -1.3143 & -0.27349 & -0.37775 & -0.35935 & -0.33325 & -0.32717 & -0.32540 & -0.32601 \\
106 & -1.5000 & -0.18897 & -0.29791 & -0.45972 & -0.46283 & -0.44226 & -0.43947 & -0.44103 \\
100 & -1.5857 & -0.14445 & -0.22425 & -0.41095 & -0.50351 & -0.50762 & -0.49468 & -0.49248 \\
96 & -1.6429 & -0.11669 & -0.17469 & -0.33494 & -0.46858 & -0.53285 & -0.53940 & -0.53784 \\
94 & -1.6714 & -0.10376 & -0.15138 & -0.28993 & -0.42548 & -0.51846 & -0.54890 & -0.55647 \\
93 & -1.6857 & -0.09758 & -0.14028 & -0.26698 & -0.39835 & -0.50133 & -0.54482 & -0.55919 \\
75 & -1.9429 & -0.02046 & -0.01231 & -0.01293 & -0.01328 & -0.00365 & 0.05945 & $\underline{0.07701}$ \\
74 & -1.9571 & -0.01798 & -0.00880 & -0.00764 & -0.00778 & 0.00234 & $\underline{0.05981}$ & 0.07563 \\
63 & -2.1143 & -0.00032 & 0.01304 & 0.02439 & 0.02956 & $\underline{0.04331}$ & 0.01392 & 0.01073 \\
58 & -2.1857 & 0.00312 & 0.01543 & $\underline{0.02737}$ & $\underline{0.03362}$ & 0.03606 & 0.00303 & 0.00208 \\
57 & -2.2000 & 0.00355 & $\underline{0.01553}$ & 0.02732 & 0.03342 & 0.03324 & 0.00153 & 0.00054 \\
50 & -2.3000 & $\underline{0.00481}$ & 0.01371 & 0.02277 & 0.02546 & 0.01252 & -0.00605 & -0.00928 \\
1 & -3.0000 & 0.00000 & 0.00000 & 0.00000 & 0.00000 & 0.00000 & 0.00000 & 0.00000 \\
\hline
\end{tabular}

Table VI. Velocity values in the $y$ direction along the horizontal line $(y=-1.0)$ in Mesh 2.

\begin{tabular}{lcrrrrrrr}
\hline & & \multicolumn{7}{c}{$R e$} \\
\cline { 3 - 9 } Grid pt. no. & \multicolumn{1}{c}{$x$} & \multicolumn{1}{c}{100} & \multicolumn{1}{c}{200} & \multicolumn{1}{c}{500} & \multicolumn{1}{c}{1000} & 2000 & 3500 & \multicolumn{1}{c}{5000} \\
\hline 201 & 1.1547 & 0.00000 & 0.00000 & 0.00000 & 0.00000 & 0.00000 & 0.00000 & 0.00000 \\
195 & 1.0854 & -0.14663 & -0.20538 & -0.33354 & -0.45622 & -0.55547 & -0.60292 & -0.61850 \\
193 & 1.0623 & -0.18510 & -0.25872 & -0.40554 & -0.51876 & -0.57957 & -0.58979 & -0.58657 \\
190 & 1.0277 & -0.23363 & -0.32360 & -0.47331 & -0.54549 & -0.54712 & -0.52833 & -0.52041 \\
186 & 0.9815 & -0.28188 & -0.38138 & -0.49887 & -0.50832 & -0.47690 & -0.46718 & -0.47034 \\
180 & 0.9122 & -0.32187 & -0.41260 & -0.45508 & -0.42435 & -0.41400 & -0.42254 & -0.42970 \\
175 & 0.8545 & -0.33011 & -0.40093 & -0.39707 & -0.37604 & -0.38205 & -0.39068 & -0.39628 \\
101 & 0.0000 & 0.10458 & 0.09033 & 0.05136 & 0.04008 & 0.03387 & 0.03207 & 0.03184 \\
60 & -0.4734 & $\underline{0.18995}$ & 0.26513 & 0.28742 & 0.26286 & 0.25367 & 0.25191 & 0.25307 \\
58 & -0.4965 & 0.18981 & $\underline{0.26566}$ & 0.29968 & 0.27459 & 0.26479 & 0.26294 & 0.26407 \\
42 & -0.6813 & 0.17807 & 0.23822 & $\underline{0.35731}$ & 0.37510 & 0.35809 & 0.35375 & 0.35480 \\
33 & -0.7852 & 0.16183 & 0.20468 & 0.32956 & $\underline{0.40430}$ & 0.41568 & 0.41038 & 0.40969 \\
27 & -0.8545 & 0.14537 & 0.17678 & 0.28510 & 0.38345 & $\underline{0.43522}$ & 0.44616 & 0.44862 \\
24 & -0.8891 & 0.13501 & 0.16088 & 0.25700 & 0.35794 & 0.42878 & $\underline{0.45412}$ & 0.46214 \\
22 & -0.9122 & 0.12723 & 0.14946 & 0.23645 & 0.33563 & 0.41633 & 0.45197 & 0.46503 \\
1 & -1.1547 & 0.00000 & 0.00000 & 0.00000 & 0.00000 & 0.00000 & 0.00000 & 0.00000 \\
\hline
\end{tabular}




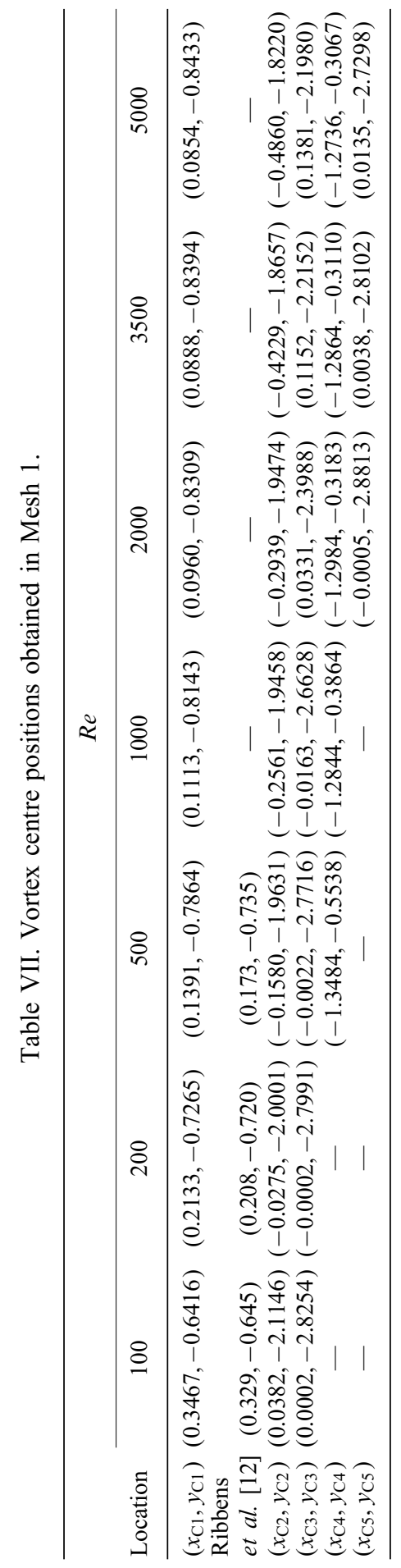

Copyright (c) 2006 John Wiley \& Sons, Ltd. 

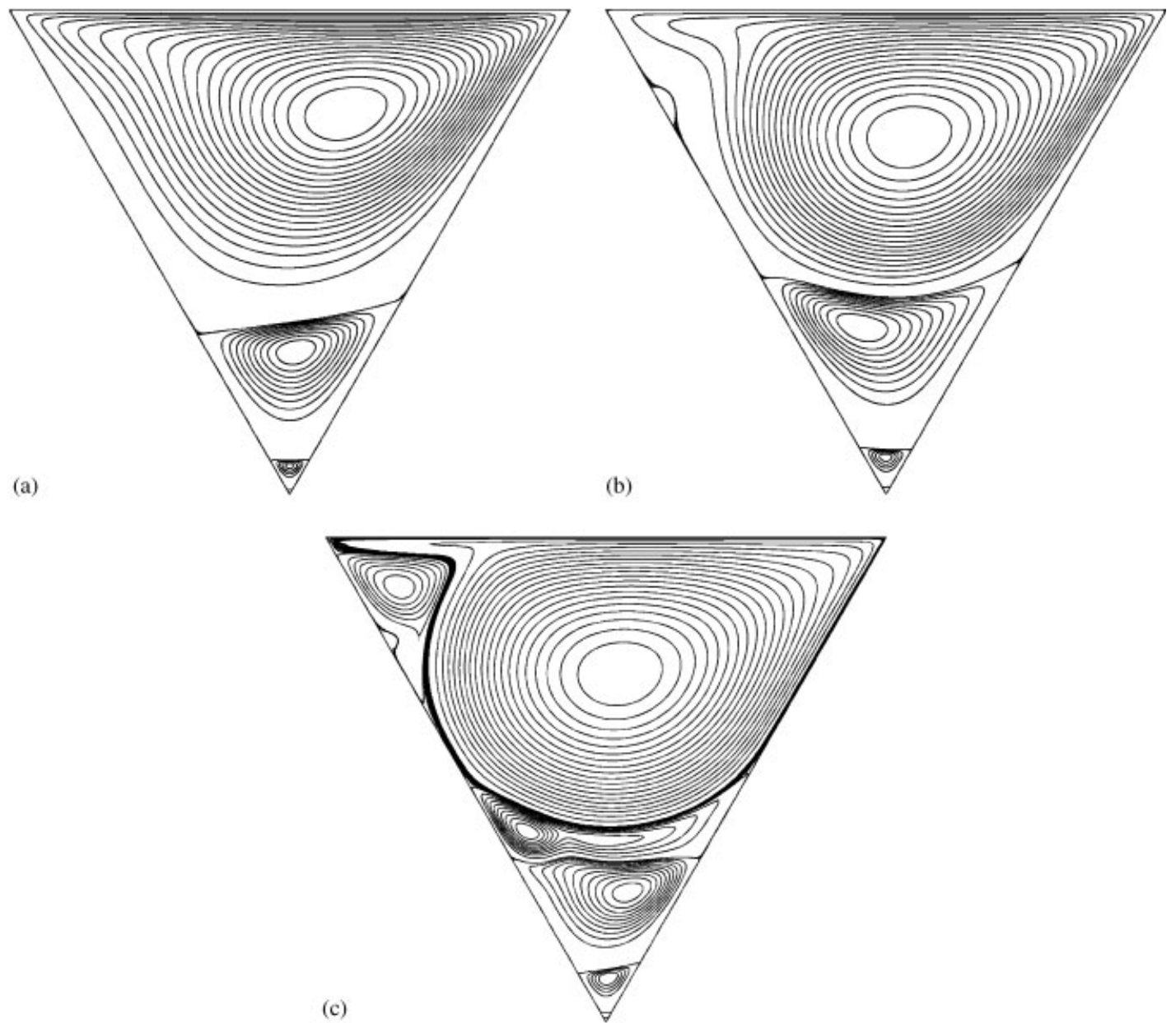

Figure 18. Streamline patterns in Mesh 1: (a) solution for $R e=100$; (b) solution for $R e=500$; and (c) solution for $R e=5000$.

In Figure 20(a), as in the square-cavity flow problem, the centre of the primary vortex moves toward the geometric centre of the cavity as the Reynolds number increases. On the contrary, the secondary eddies under the primary vortex first appear around the cavity centre and then move right or left with the increase in the Reynolds number as shown in Figures 20(b), (c) and (e). This figure also implies that more eddies will appear near the stagnant corner at higher Reynolds numbers; but a finer mesh need be used to capture those tiny eddies.

\section{CONCLUSIONS}

In this paper we presented an FCBI scheme for use with triangular grids in the solution of the Navier-Stokes equations at low and high Reynolds numbers. The emphasis in the FCBI procedure 

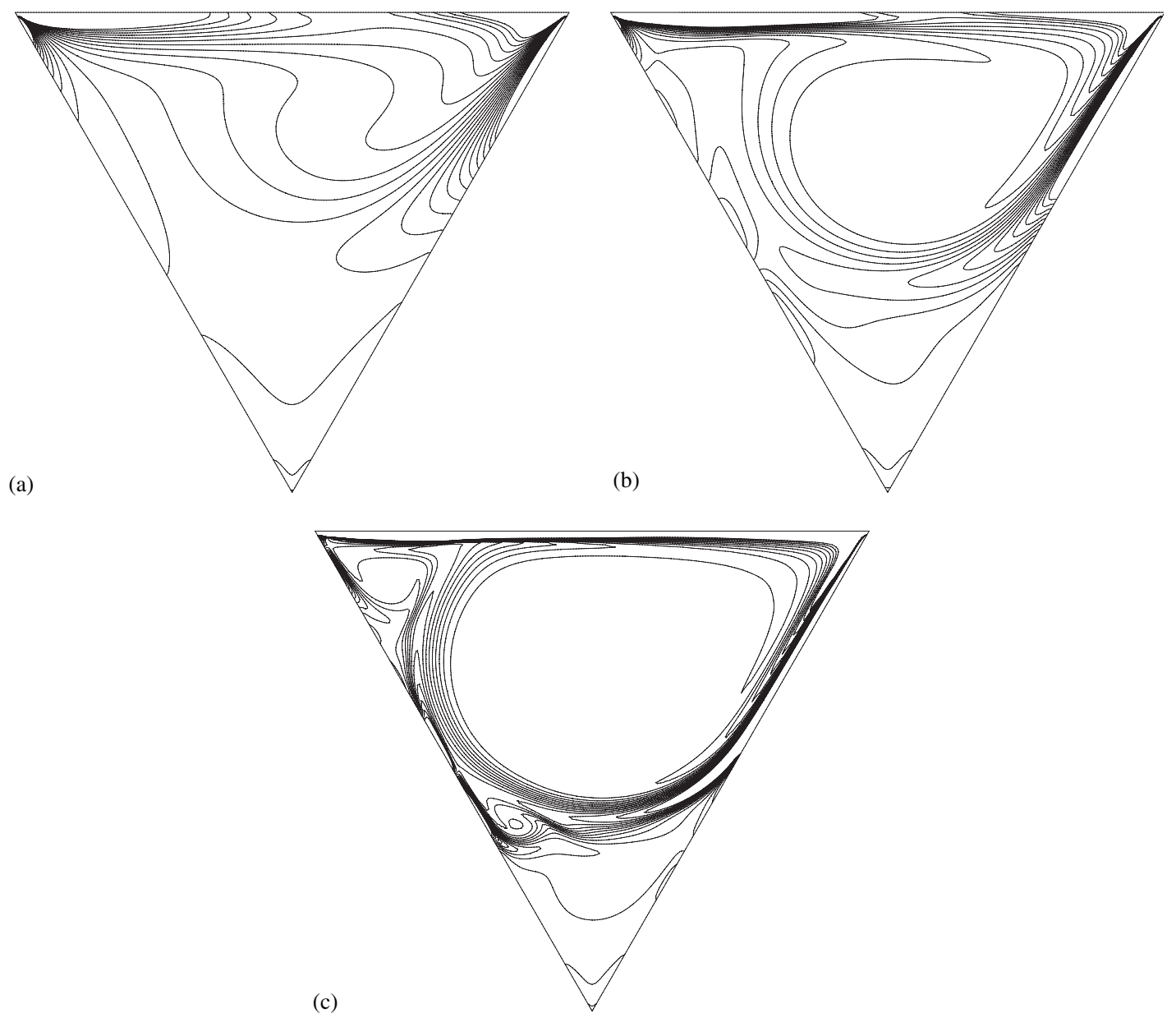

Figure 19. Vorticity contours in Mesh 1: (a) solution for $R e=100$; (b) solution for $R e=500$; and (c) solution for $R e=5000$.

is on stability and reasonable accuracy even when rather coarse meshes are used. In the case of triangular discretizations, we also want that property to hold when completely unstructured meshes are employed. This is difficult to achieve, but a reasonable research aim. The scheme presented in the paper is spatially isotropic (which is important for general applications) and showed good stability and accuracy in the test problems solved. Some detailed results are given for the flow fields in a driven square-cavity problem and in a driven triangular-cavity problem.

The scheme was presented and tested for two-dimensional solutions, but in principle the given procedure can also directly be developed for three-dimensional analyses. Of course, further studies of the scheme, including the numerical effectiveness, for two-dimensional solutions are needed. These studies might also result in improvements of the procedure, and for three-dimensional solutions, the scheme needs to be still implemented, thoroughly tested and analysed. Finally, a mathematical analysis of the given scheme would be very valuable. 

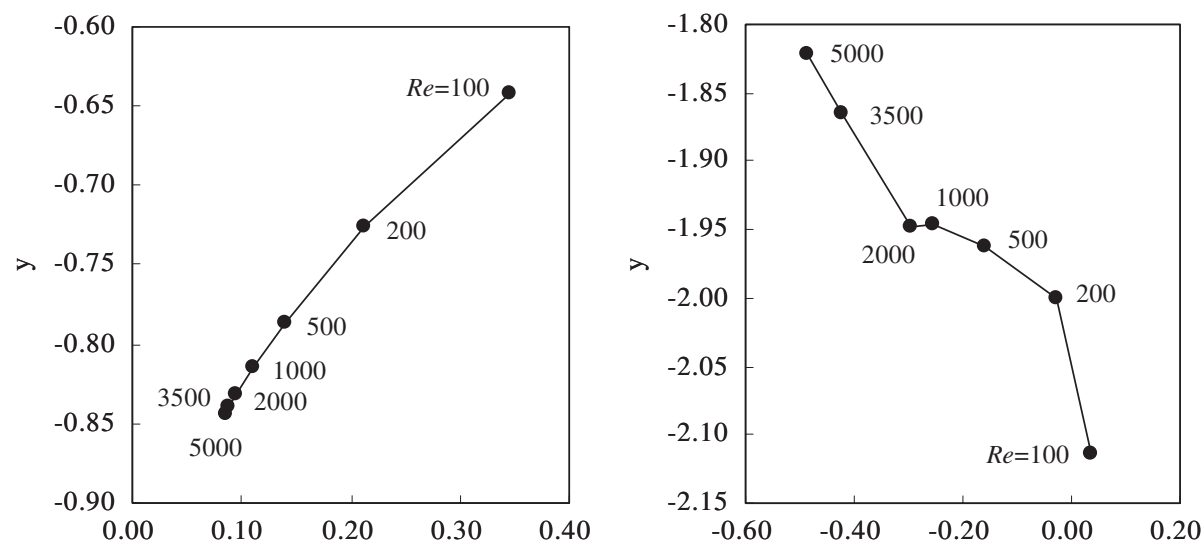

(a)

(b)
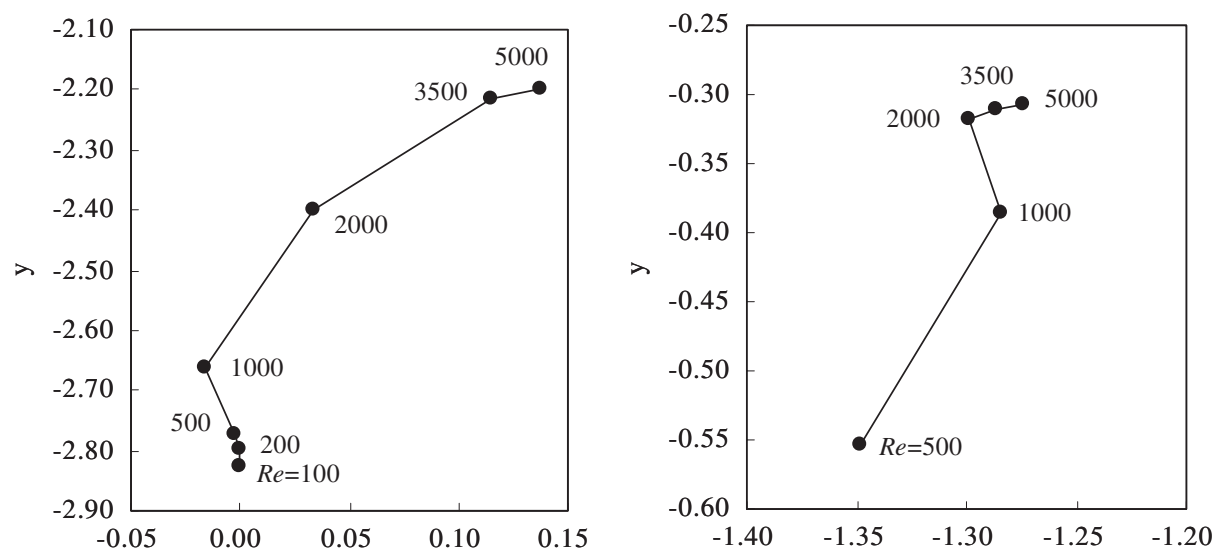

(c)

$\mathrm{x}$

(d)

$\mathrm{x}$

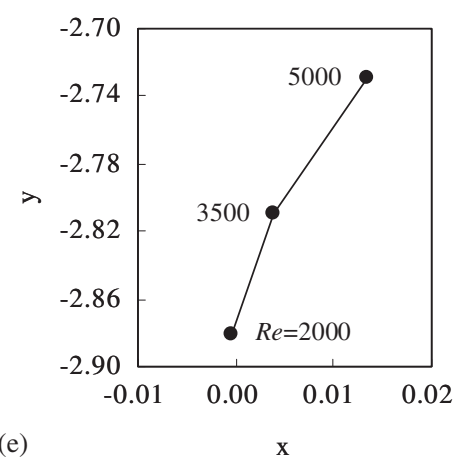

Figure 20. Variation of the vortex centre positions in Mesh 1: (a) $\left(\mathrm{x}_{\mathrm{C} 1}, \mathrm{y}_{\mathrm{C} 1}\right)$; (b) $\left(\mathrm{x}_{\mathrm{C} 2}, \mathrm{y}_{\mathrm{C} 2}\right)$; (c) $\left(\mathrm{x}_{\mathrm{C} 3}, \mathrm{y}_{\mathrm{C} 3}\right) ;(\mathrm{d})\left(\mathrm{x}_{\mathrm{C} 4}, \mathrm{y}_{\mathrm{C} 4}\right)$; and $(\mathrm{e})\left(\mathrm{x}_{\mathrm{C} 5}, \mathrm{y}_{\mathrm{C} 5}\right)$. 


\section{ACKNOWLEDGEMENTS}

We are grateful for the financial support of H. Kohno by the Ministry of Education, Culture, Sports, Science and Technology (MEXT), Japan.

\section{REFERENCES}

1. Bathe KJ (ed.). Computational fluid and solid mechanics. Proceedings of the Second MIT Conference on Computational Fluid and Solid Mechanics 2003. Elsevier: Amsterdam, 2003.

2. Bathe KJ (ed.). Computational fluid and solid mechanics. Proceedings of the Third MIT Conference on Computational Fluid and Solid Mechanics 2005. Elsevier: Amsterdam, 2005.

3. Gresho PM, Sani RL. Incompressible Flow and the Finite Element Method. Wiley: New York, 2000.

4. Drikakis D, Rider W. High-Resolution Methods for Incompressible and Low-Speed Flows. Springer: Berlin, 2005.

5. Bathe KJ, Pontaza JP. A flow-condition-based interpolation mixed finite element procedure for higher Reynolds number fluid flows. Mathematical Models and Methods in Applied Sciences 2002; 12(4):525-539.

6. Bathe KJ, Zhang H. A flow-condition-based interpolation finite element procedure for incompressible fluid flows. Computers and Structures 2002; 80:1267-1277.

7. Kohno H, Bathe KJ. Insight into the flow-condition-based interpolation finite element approach: solution of steady-state advection-diffusion problems. International Journal for Numerical Methods in Engineering 2005; 63:197-217.

8. Bathe KJ, Zhang H. Finite element developments for general fluid flows with structural interactions. International Journal for Numerical Methods in Engineering 2004; 60:213-232.

9. Bathe KJ. Finite Element Procedures. Prentice-Hall: Englewood Cliffs, NJ, 1996.

10. Hendriana D, Bathe KJ. On upwind methods for parabolic finite elements in incompressible flows. International Journal for Numerical Methods in Engineering 2000; 47:317-340.

11. Ghia U, Ghia KN, Shin CT. High-Re solutions for incompressible flow using the Navier-Stokes equations and a multigrid method. Journal of Computational Physics 1982; 48:387-411.

12. Ribbens CJ, Watson LT. Steady viscous flow in a triangular cavity. Journal of Computational Physics 1994; 112:173-181.

13. Jyotsna R, Vanka SP. Multigrid calculation of steady, viscous flow in a triangular cavity. Journal of Computational Physics 1995; 122:107-117. 